\title{
Spatial selection via feature-driven inhibition of distractor locations
}

\author{
NICHOLAS J. CEPEDA \\ University of Illinois at Urbana-Champaign, Urbana, Illinois \\ and \\ KYLE R. CAVE, NARCISSE P. BICHOT, and MIN-SHIK KIM \\ Vanderbilt University, Nashville, Tennessee
}

\begin{abstract}
The allocation of spatial attention was measured with detection probes at different locations. Response times were faster for probes at the location of the target digit, which subjects reported, than at the locations of distractor digits, which they ignored. Probes at blank locations between stimuli produced fast responses, indicating that selection was accomplished by inhibiting distractor locations but not other areas. Unlike earlier studies using location cuing with simpler stimuli, these experiments showed no attentional differences across horizontal or vertical midlines. Attention varied little with distance from the target, although blank locations far from the target were somewhat less attended than were those near the target, and attention was only slightly affected by expectations for stimulus location. This task demonstrates a form of feature-driven spatial attention, in which locations with objects lacking target features are inhibited.
\end{abstract}

In the course of visual processing, some regions of the visual field receive more thorough processing than do others. Presumably, when multiple objects appear simultaneously, information from each object can interfere with the processing of the others, and this selection process, or spatial attention, limits this interference by favoring the locations of some objects over others. One early demonstration of spatial attention came from experiments using spatial cuing. They established that the presentation of a cue indicating a particular location in the visual field could draw attention to that location, so that subjects responded to a subsequent stimulus at that location more quickly than to a stimulus at some other, uncued location (Eriksen \& Hoffman, 1974; Posner, Nissen, \& Ogden, 1978; Posner, Snyder, \& Davidson, 1980).

These cuing experiments stimulated a series of studies that attempted to produce more complete maps of the allocation of spatial attention across the visual field. In these later studies, the relative positions of cue and test stimulus were systematically varied. For instance, Hughes and

M.-S. Kim is currently at the Department of Psychology, Yonsei University, Seoul, 120-749, Korea. This work was supported in part by Grant P30-EY08126 from NEI awarded to the Vanderbilt Vision Research Center. Data from Experiments 1 and 2 were presented at the meeting of ARVO, May 1995, in Ft. Lauderdale, FL. Thanks to Ben Phillips, Vik Barad, and Tarsicio Duran for help in conducting experiments, and to Volker Thoma, Matthew Heisler, Art Kramer, MaryLou Cheal, Carolyn Backer Cave, Roger Remington, Lynn Zimba, and an anonymous reviewer for comments. Thanks also to Paul Russell and Tom Birkett for providing software to control the Macintosh response keys and a design for the response key interface, and to Lonny Shimp for building the response keys. Correspondence should be addressed to K. R. Cave, Department of Psychology, Vanderbilt University, 301 Wilson Hall, Nashville, TN 37240 (e-mail: kyk.r.cave@vanderbilt.edu).
Zimba $(1985,1987)$ showed that responses were uniformly fast whenever cue and target were in the same hemifield, and responses uniformly slow when they were in different hemifields. They found similar results whether they tested left versus right or top versus bottom. If attention always activated an entire hemifield, though, it would be of little help in eliminating interference from distractor objects. A mechanism that selects an entire hemifield or quadrant will generally be unable to select a target location while excluding distractor locations.

Visual selection has been somewhat more narrowly distributed in some other cuing experiments. One study, by Downing and Pinker (1985), included cues and test stimuli at each of 10 different locations along the horizontal midline. Rather than selection of an entire hemifield, they found a gradient of attention surrounding the cued location. Response time (RT) for detecting the test stimulus increased gradually with distance between the expected (cued) location and the actual test stimulus location. Their data were somewhat unclear, but they suggested that the size of the cuing effect depended not on the physical distance between cue and stimulus but on the "cortical distance" between the two points. Since more visual cortex is devoted to processing the center of the visual field, distance effects were much larger when the cue and test stimulus were near the center and smaller when they were in the periphery. The largest effects occurred when the two points were on opposite sides of the vertical midline.

Rizzolatti, Riggio, Dascola, and Umiltà (1987) also found slower responses with longer distances between a cue and test stimulus within the same quadrant, as Downing and Pinker would have predicted. They also found an 
additional cost when cue and target were on opposite sides of either the horizontal or the vertical midline, and because their stimuli were iocated peripherally, they concluded that the extra cost was not due to cortical magnification. (Rizzolatti et al. claimed that delays associated with cuing, including the extra time associated with crossing the midline, reflect the time necessary to program eye movements.)

One important difference among these studies is that Downing and Pinker (1985) and Rizzolatti et al. (1987) marked each of their possible target locations with a square, whereas Hughes and Zimba's $(1985,1987)$ stimuli appeared on a relatively empty screen. Extra contours in the display may serve as weak distractors, interfering somewhat with detection of the targets and prompting the subjects to employ more narrowly focused spatial selection. When Zimba and Hughes (1987) replicated Downing and Pinker's experiment with 10 squares marking the 10 possible target locations, they found distance effects as well. In other experiments, Zimba and Hughes found that a distractor spol of light in the unattended hemifield elicited attentional inhibition that was focused on a region with a radius of about $2^{\circ}$ around the spot. These experiments taken together suggest that spatial attention can be applied more specifically when the displays include other stimuli. Lavie and Tsal (1994) also found important differences in visual selection depending on the presence of distractors. After reviewing a large number of different experiments, they concluded that spatial selection occurs only under heavy perceptual load. Further evidence comes from a study by Lavie (1995), who used an interference task to demonstrate stronger selection with heavy load.

Mangun and Hillyard (1988) demonstrated the distribution of attention after iocation cuing by measuring attentional enhancement of ERP signals rather than RTs. In some cases, they found that attentional effects were strongest at the cued location, weakest at a location far from the cue, and in-between at an intermediate location. The pattern they found could be attributed to either midline effects or to distance, because the location far from the cue was in the opposite hemifield, and the intermediate location was on the midline.

Although attention was somewhat more spatially restricted in Downing and Pinker's (1985) experiment than in Hughes and Zimba's $(1985,1987)$ original tests, it was still too broadly distributed to prevent interference from distractors in complex scenes. If one of the main purposes of visual attention is to prevent interference between information from different objects, then the specificity with which locations are selected may not matter as much in these tasks. The displays include few visual objects that might be confused with the targets. Even in Downing and Pinker's displays with the squares at each possible stimulus location, there was nothing in the display that could easily be confused with the test stimulus. Perhaps these tasks can be performed fairly well whether or not attention is applied in a focused way. Attention may be allocated with more spatial specificity when there is a larger threat of interference from distractors. Also, a selection mechanism that is constrained by the boundaries between quadrants would be of limited use in most complex scenes, because target and distractor objects will usually not be conveniently arranged on opposite sides of these boundaries. When spatial selection is required to prevent interference from distractors that are easily confused with the target, it might be less affected by the presence of quadrant boundaries.

The cuing experiments described above measured how attention is driven by expectations of an upcoming stimulus' location or, in the case of peripheral cuing, the perceptual differences between the cue and the rest of the display. There may be limits on how specifically attention can be targeted on the basis of these factors. Once a stimulus has appeared, however, attention might be allocated more specifically according to the locations of relevant features of the target and distractors (for evidence from neuroscience, see Treue \& Maunsell, 1996). The presence of distractors in the stimulus may also elicit a more spatially restricted selection. Thus, more restricted spatial selection may more easily be demonstrated in experiments that measure how spatial attention is allocated spontaneously in the course of processing complex stimuli that include distractors. Previous experiments have used two successive tasks to measure attentional allocation (Banks, Kouwabunpat, \& Ciranni, 1992; Cave \& Pashler, 1995; Cave \& Zimmerman, 1997; Hoffman \& Nelson, 1981; Hoffman, Nelson, \& Houck, 1983; Kim \& Cave, 1995; LaBerge, 1983; Luck, Fan, \& Hillyard, 1993; Sagi \& Julesz, 1986; Tsal \& Lavie, 1988, 1993). In these experiments, subjects first focused their attention on a target at one location while ignoring distractors at other locations. Subjects then performed better at detecting a subsequent stimulus when it appeared at or near the location formerly occupied by the target than when it was at a location that had been occupied by a distractor (see Cave \& Bichot, in press, for a brief review of these studies).

Klein (1988) used such a combination of visual search and a probe task to demonstrate inhibition of return during conjunction search. His conjunction-search subjects responded more slowly to probes at locations that had contained distractors than to those at blank locations. Masking was ruled out because the difference between distractor and blank locations was smaller in a less attentionally demanding parallel search task. Subsequent studies (Klein \& Taylor, 1994; Wolfe \& Pokorny, 1990) found slower RTs for distractor locations in both search tasks, and the difference was attributed to masking rather than attentional inhibition. These studies show how interpretation of probe responses can be complicated by masking.

The experiments reported in this paper also measured attentional allocation in response to more complex stimuli, using techniques similar to those in Cave (1995), Cave and Zimmerman (1997), and Kim and Cave (1995). In those earlier studies, subjects were engaged in two tasks in each trial. For the primary task in Cave (1995), they viewed a display with two digits: one was a target that they 
were to report, and the other was a distractor that they were to ignore. The target and distractor were designated by color. The second task consisted of detecting a small probe stimulus that sometimes appeared immediately after the primary stimulus. The probe could appear at the location formerly occupied by either the target digit or the distractor digit. RTs to the probe were faster when it appeared at the target location, indicating that attention was favoring the target location over the distractor location and that detection of a probe at the target location was thus facilitated relative to one at the distractor location. Experiment 3 of Cave's (1995) study also probed blank locations near the target and distractor, and some spread of attentional effects to areas near the digit locations was detected, at least in some cases. The spatial nature of the selection process measured by these probes was underscored by Cave and Zimmerman's experiment, which demonstrated that distractor locations near a target were inhibited more than distractor locations far from the target.

Just as Downing and Pinker (1985) and Hughes and Zimba (1985) explored the allocation of spatial attention across the visual field in response to a cue, the experiments described in this paper explored spatial attention allocation across the visual field during the processing of a stimulus composed of a target and distractor digits. The distractors in the primary task can potentially interfere with target identification, and thus spatial attention may be more important in this task than in cuing tasks. The present experiments tested the spatial distribution of attention when distractors are present and tested how attentional effects spread from target and distractor locations to the regions around them. The probe RTs revealed selection mainly in the form of inhibition of distractor locations relative to the background, which spread only slightly to the blank regions between distractors. They also showed no attentional differences on opposite sides of the horizontal and vertical midlines that separate the visual field quadrants.

\section{EXPERIMENT 1 Attention Across Distances and Midlines}

In Experiment 1, the primary stimulus, consisting of distractor digits and a single target digit of a different color, was presented first. The primary task was to identify the target digit, although subjects did not report its identity immediately. The primary task caused subjects to select the target location over the distractor locations. After a short interval, the primary stimulus disappeared, and a probe was presented at the target location, a distractor location, or one of the blank locations between digits. RTs to detect the probes were measured, and these RTs indicated the amount of attentional facilitation or inhibition allocated to the probe location. Attention might be allocated more or less quickly depending on amount of practice and on the difficulty of the primary task. Therefore, the time between the primary stimulus and the probe (stimulus onset asynchrony, SOA) was varied.
Probes were presented on only half the trials, and subjects did not know on any given trial whether or not a probe would appear.

\section{Method}

\section{Subjects}

Twenty students at Vanderbilt University participated in this experiment. All were fulfilling an introductory psychology course requirement. None knew the purpose of the experiment beforehand. All subjects reported normal or corrected-to-normal vision, including normal color perception. Most subjects finished in less than $1 \mathrm{~h}$. Ten searched for a red target among green distractors, and 10 searched for green among red. One subject could not accurately discriminate colors on the screen and was replaced by a new subject.

\section{Apparatus}

The experiment was conducted on three Macintosh IIsi computers with 13-in. AppleColor (Trinitron) monitors. The subjects responded via the Macintosh keyboard.

\section{Stimuli}

Each primary stimulus contained a single digit of the target color and three digits of the distractor color. The red and green used for the digits were approximately matched for luminance with a photometer (C.I.E. relative photopic luminosity curve), although differences across the three monitors were not corrected. The background was white. Distance from subject to screen was approximately $58 \mathrm{~cm}$. Each digit was $11 \mathrm{~mm}\left(1.1^{\circ}\right.$ visual angle $)$ tall $\times 8 \mathrm{~mm}\left(0.8^{\circ}\right.$ visual angle) wide. The target and distractor digits were randomly selected from the digits between 2 and 9 , with replacement. All four digits were equally spaced on an imaginary circle with a diameter of $120 \mathrm{~mm}\left(11.8^{\circ}\right.$ visual angle) around fixation (see Figure 1). One digit appeared in each quadrant. For half the subjects in each group, each digit was positioned $22.5^{\circ}$ rotationally in the clockwise direction from the nearest horizontal or vertical midline; for the other half, they were $22.5^{\circ}$ in the counterclockwise direction from the midline. Throughout the experiment, there was a black fixation cross at the center of the screen.

\section{Procedure}

The sequence of displays in a single trial is shown in Figure 2. Throughout each trial, the subjects fixated on a cross in the center of the screen. On each trial, the primary stimulus, with the target

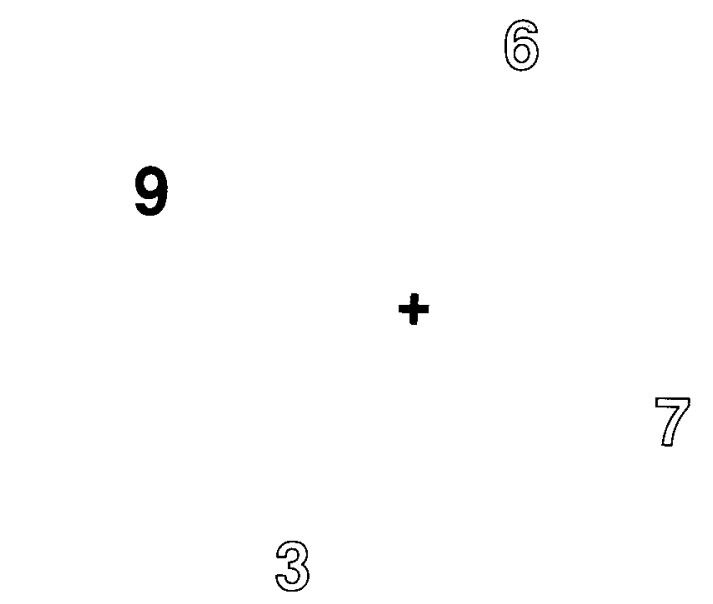

Figure 1. An example of the display used in Experiment 1. The target color is represented by black, and the distractor color is represented by white. 


\section{Regular Trial (50\%)}
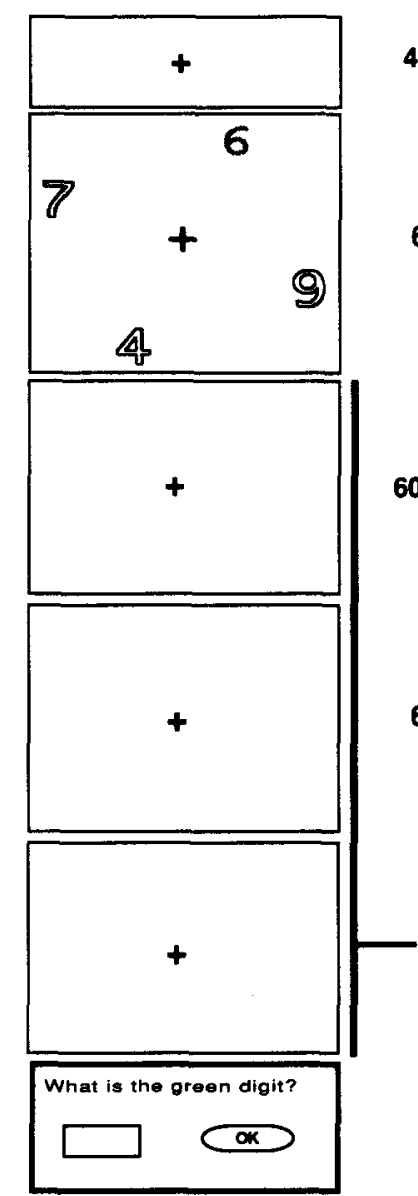

400 msec

Probe Trial (50\%)

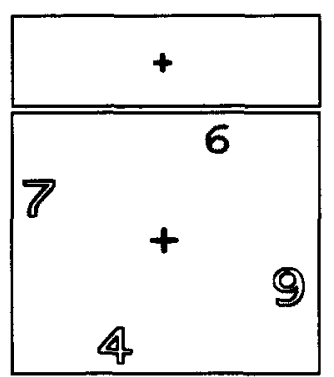

60,90 , or 120

msec ISI

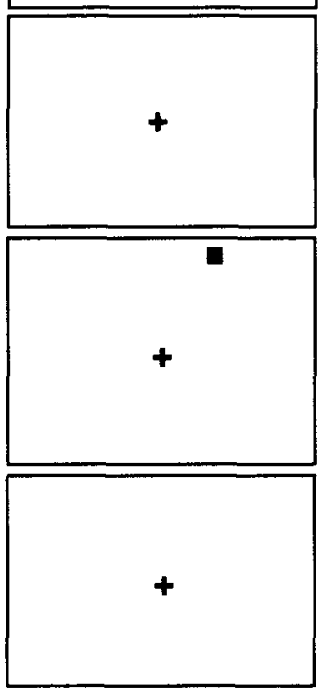

What is the green digit?

1400

msec

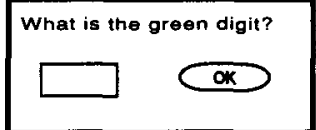

Figure 2. The sequence of events within a trial for Experiment 1, for both probe and no-probe trials. The target digit is drawn solid here, and distractors are drawn in outline. All digits were solid in the experimental display.

digit and three distractor digits, appeared for $60 \mathrm{msec}$. The target digit was positioned in a randomly selected quadrant. Then, after a $1,400-\mathrm{msec}$ interval, a question appeared on the screen asking the subject to name the target digit (primary task). The subjects responded by pressing the correct digit on the keyboard. They were instructed that speed was not important for this task and that they could take as long as needed to respond correctly.

The 1,400-msec delay between primary stimulus and response provided time for the probe task. Half the trials were probe trials, and half were no-probe trials. In the no-probe trials, nothing other than the fixation cross appeared during these $1,400 \mathrm{msec}$. In the probe trials, following a delay of 120,150 , or $180 \mathrm{msec}$ after the digits first appeared, a solid black square was presented for $60 \mathrm{msec}$. The square was $5.5 \mathrm{~mm}$ on each side $\left(0.55^{\circ}\right.$ visual angle), and its center was always on the same imaginary circle on which the digits' centers were positioned. The probe appeared equally often at each of eight locations, which were $22.5^{\circ}$ clockwise or $22.5^{\circ}$ counterclockwise from a midline. In other words, it was either at a position formerly occupied by one of the digits or at a blank position halfway between two digits. Probe position varied randomly within blocks. The subjects were instructed to press the space bar as quickly as possible after the probe appeared. Time between the dis- play of digits and appearance of the digit question remained constant for probe and no-probe trials.

There were 12 blocks of 64 trials each, making a total of 16 probe trials for each combination of SOA and probe position relative to target position. The different trial types were randomly distributed across blocks. After each block, the computer prompted the subjects to take a break. Each subject completed a block of 64 practice trials before the experiment began, and the subjects were allowed more practice if they desired it.

\section{Results}

Performance in the primary task was very good for most subjects. Average error rate across all subjects was below $3 \%$, and all but 2 subjects averaged less than $3 \%$. The remaining 2 subjects had error rates of $25 \%$ and $7 \%$, and they were in the green-target and red-target groups, respectively.

The subjects were also very accurate in detecting probes. The mean error rate for the probe task was only $0.4 \%$. Error rates were calculated separately for each com- 
bination of the three SOAs and the eight probe locations. Of these 24 means, 7 were $0 \%$, and the highest was only $1.1 \%$. We judged these error rates to be too low to show any meaningful effects, and we did not analyze them.

Trials in which the subjects made an error in the primary task were not included in the probe RT analyses. (Error trials were excluded in Experiments 2 and 3 as well.) Extreme values were removed from the probe RTs before they were analyzed, to eliminate the trials in which the subjects prematurely responded to a probe or the trials in which the subjects did not respond to the probe until after an unusually long delay. The trimming procedure iteratively removed the highest and/or lowest RT in a condition if it was more than 3.5 standard deviations $(S D s)$ from the mean of the remaining RTs. The means were calculated and the trimming done separately for every combination of subject, SOA, and probe position. Fewer than $4 \%$ of all RTs were removed in trimming. Figure 3 shows mean RTs to the probes at target locations, distractor locations, blank locations near the target, and blank locations far from the target.

The data were analyzed in three parts. First, probe RTs at the different digit locations were compared in order to demonstrate selection of the target location over the distractor locations. Second, blank locations were compared with one another in order to determine whether attention spread from the digits to neighboring blank locations. Third, distractor locations were compared with blank locations in order to see whether all locations without a target received the same attentional treatment. SOA and color were included as factors in these analyses, but the few effects and interactions involving these factors did not seem to affect the overall conclusions, and they will not be reported.

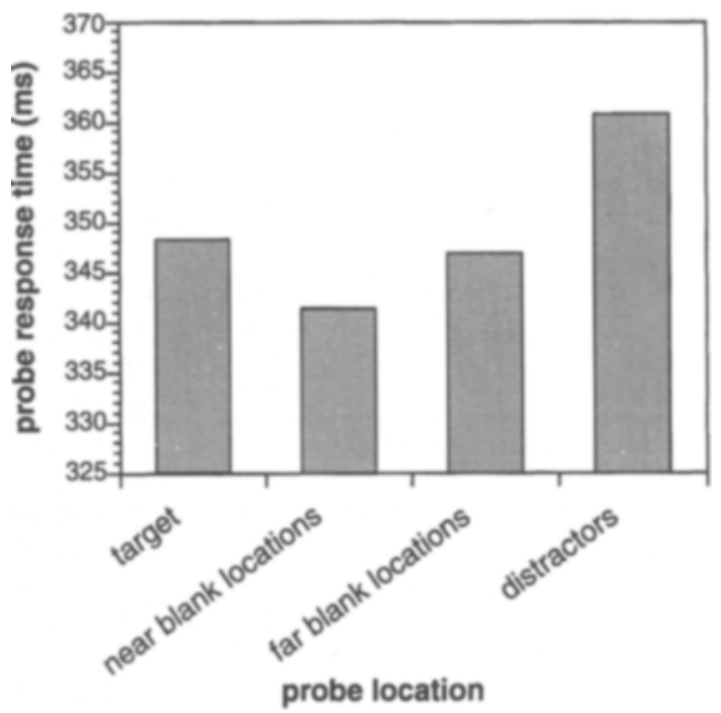

Figure 3. Probe response times for Experiment 1.

\section{Digit Locations}

Probe RTs from only the digit locations were submitted to a repeated measures analysis of variance (ANOVA), with probe position as a factor. The four possible digit positions were target, quadrant across the midline nearest the target, quadrant across the other midline from the target, and quadrant across both midlines from the target. ${ }^{1}$ A planned contrast showed that the target location produced faster RTs than did the three distractor locations $[F(1,54)=8.59, p<.01]$. As in previous experiments (Cave, 1995; Cave \& Zimmerman, 1997; Kim \& Cave, 1995), the probe technique demonstrates that spatial attention is allocated to favor the target location over distractor locations.

Another analysis tested for differences among the three distractor locations, which would be expected if the effects of attention decrease with distance from the target or if attention is allocated only to one hemisphere or quadrant. We employed a Tukey test to perform all three of the possible pairwise comparisons of the three distractor digit locations. No difference in probe RT was found between any pair of distractor locations, suggesting that all distractor locations were equally inhibited, regardless of their distance from the target, or that the target location was facilitated, or both.

\section{Blank Locations}

The second set of analyses tested for attentional differences among the blank locations. They were based on a repeated measures ANOVA, with probe location as the only within-subjects factor. In the stimulus displays, there were four blank locations that could be probed, each sharing a quadrant with one of the four digit locations. Within these analyses, each blank location was classified according to the location of its quadrant relative to the target (target quadrant, quadrant across the midline nearest the target, etc.).

One planned contrast tested whether the two blank locations near the target received more facilitation or inhibition than the two locations farther from the target. This contrast was significant $[F(1,54)=4.367, p<.05]$. Perhaps facilitation spreads from the target location to neighboring blank locations, and/or inhibition spreads from distractor locations. The blank locations with the target on one side and a distractor on the other would then be relatively more facilitated than those between two distractors.

A second set of planned contrasts tested for midline effects between positions that were equally distant from the target. The first contrast compared the two blank locations near the target: one that was in the same quadrant as the target, and one that was across either the horizontal or the vertical midline. The other compared the two farther positions: one that was in the quadrant opposite the target and, thus, across both midlines, and one that was across only one midline from the target. Neither contrast was significant. 


\section{Digit Versus Blank Locations}

The third set of analyses tested for differences between digit locations and blank locations. These differences could reflect attentional differences, although they might be due to masking, as discussed in Experiment 4. Two separate tests were done comparing these locations. The first tests compared the target location with the two blank locations next to the target in order to see whether the target location received attentional treatment different from that received by the nearby blank areas. A repeated measures ANOVA included three probe locations: target, neighboring blank location within target quadrant, and neighboring blank location across a midline from target quadrant. The target location mean was somewhat slower than the two blank location means combined, although a planned contrast was not significant $[F(1,36)=2.7$, $p>.10]$. Ignoring the possibility of masking for now, the target location generally seems to receive the same attentional treatment as the blank locations near it. If there is any difference, it is in the form of target inhibition, perhaps related to inhibition of return (see Posner \& Cohen, 1984).

The second test compared the three distractor locations with the three blank locations in the distractor quadrants (there was one blank location and one distractor location in each of the three quadrants). This test involved a repeated measures ANOVA, with both digit versus blank position and quadrant as factors. Probes at the blank locations in distractor quadrants generally elicited faster responses than did probes at the digit locations in the same quadrants $[F(1,18)=25.16, p<.001]$. The differences between digit and blank locations appeared in all three of the quadrants tested, because there was not a significant interaction between quadrant and the digit/blank factor $(F<1.0)$.

\section{Discussion}

The RT patterns show that the location with the target color is selected over locations with the distractor color and that this selection then affects the speed of response to the probe square that appears after the digits have disappeared. In Experiment 1, as in the experiments of Cave and Pashler (1995), the task was designed to encourage selection by color. The location of the stimulus was irrelevant to the response. Yet, in all these experiments, the subjects used location-based attention to select the target over distractors. Separate analyses show that both red and green target locations produce faster responses than do distractor locations. Thus, the difference in RTs between target and distractor locations cannot be attributed to some difference between the red and green stimuli, such as a difference in luminance or in masking. The RT differences must instead reflect attentional selection.

Before the primary stimulus appeared, the subjects did not know the target location and thus could not initiate an eye movement there. For the shortest SOA, only $180 \mathrm{msec}$ passed between the onset of the target and the offset of the probe. The subjects should have been unable to saccade to the target location before the probe disappeared. There was a significant target advantage for green targets at this SOA, which must have been due to attentional changes and not changes in eye position. A similar target advantage appeared with red targets at later SOAs, which also probably reflects spatial attention. If the subjects had violated the instructions and moved their eyes to the target at these longer SOAs, we might have expected that their responses for distractor locations nearer the target would be faster than those farther from the target.

The pattern of RTs suggests that selection is accomplished in this task by inhibiting the distractor locations relative to the target and to the background. The inhibition is fairly uniform across all distractor locations. However, the difference between distractor and blank locations could be due to masking, rather than differential attention. Experiment 4 addressed that question.

Experiment 1 showed no sign that attention is allocated differently across vertical and horizontal midlines than it is within a quadrant. Although we cannot rule out some weak midline effects not detected by this experiment, they would have to be very weak relative to the differences seen between target and distractors. This pattern differs from that found in cuing experiments, and it suggests that attention allocated in response to complex stimuli differs in important ways from attention elicited by location cues.

Although all the blank locations produce fast probe RTs, those far from the target are significantly slower than those near the target. When the analyses were performed separately for each combination of SOA and target color, this contrast was significant only for the longest SOA (and only for green targets), suggesting that inhibition gradually spreads from the distractor locations to affect blank areas between them. Alternatively, this pattern may reflect attention "zooming in" to the target location over time, as proposed by Theeuwes (1995). His conclusion was motivated by an experiment measuring the interference from an onset distractor during a search task. The inhibition diminished as the onset distractor appeared farther from the target and as it appeared later than the target and other distractors. If attention were zooming in to the target in the present experiment, we would also expect RTs for distractor locations close to the target to be faster than those farther from the target, which they were not. Despite these differences, the similarities in the two patterns suggest a general tendency for visual selection to first act against locations farther from the target.

\section{EXPERIMENT 2 Expected Versus Unexpected Locations}

If inhibition is applied to each distractor location, as the results of Experiment 1 suggest, then how are the distractor locations determined so that inhibition can be po- 
sitioned to block them? Spatial attention may be centrally controlled by a mechanism that identifies distractor locations and plans a pattern of inhibition to cover each of them. If such a central mechanism retains information about stimulus locations over many trials, then it may learn where distractors are most likely to appear, and it may start allocating inhibition in advance, anticipating the upcoming stimulus. Once the stimulus appears, inhibition from the location with the target color can be removed, leaving the three distractor locations inhibited. On the other hand, attention allocation in this task may be controlled by a distributed system with a simple mechanism for each location. Each mechanism would be driven by the stimulus at its location and would be fairly independent of the selection mechanisms at other locations. A simple distributed mechanism would also be less likely to store information about previous trials and use it to guide attention. With this distributed mechanism, attention allocation would more likely depend on the properties of the stimulus rather than expectations and would be the same for digits at the expected and unexpected locations.

Experiment 2 tested for differences in the pattern of spatial attention when digits appeared at either expected or unexpected locations. Probes again appeared at target locations, distractor locations, and blank locations in between. This experiment used the same basic procedures as Experiment 1. Because the previous tests showed effects of attention allocation with both red and green targets, Experiment 2 used only green targets. Also, all probes occurred at a single SOA, $150 \mathrm{msec}$ after the primary stimulus appeared. By limiting tests to a single SOA and target color, we were able to collect more data relating to the other relevant factors. This SOA was chosen because it was in the middle of the range of SOAs that showed attention effects in Experiment 1.

\section{Method}

\section{Subjects}

Twenty-two students at Vanderbilt University participated in this experiment. All were fulfilling an introductory psychology course requirement. None knew the purpose of the experiment beforehand, and none had participated in Experiment 1. All the subjects reported normal or corrected-to-normal vision, including normal color perception. Most subjects finished in less than $1 \mathrm{~h}$.

\section{Apparatus}

This experiment was conducted with the same computers used in Experiment 1

\section{Stimuli}

In Experiment 1, all the stimuli for half the subjects had digit locations that were offset clockwise from the midlines, and all the stimuli for the other half of the subjects had digit positions offset counterclockwise. In Experiment 2, each subject saw digits with a clockwise offset on some trials and a counterclockwise offset on others. For approximately half the subjects, the digits were offset clockwise on $75 \%$ of the trials, and, thus, these locations were the "expected locations" for these subjects. The remaining $25 \%$ of the trials had digits at the counterclockwise offsets, or the "unexpected locations." Figure 4 illustrates the relationships between the ex-

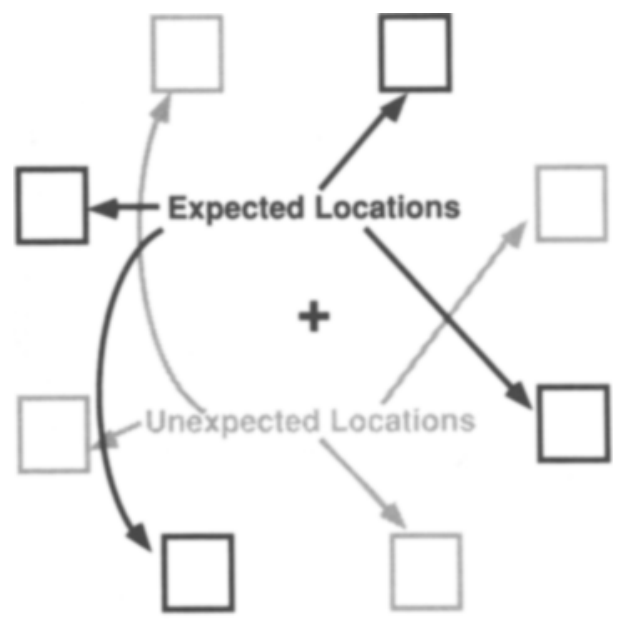

Figure 4. The configuration of the four expected and four unexpected locations. For half the subjects, the two groups of locations were reversed.

pected and unexpected locations. For the other half of the subjects, expected and unexpected locations were reversed. In a given trial, the four digits were either all at expected locations or all at unexpected locations. The target digits were always green, and the distractors were always red. Except for these changes, the stimuli for Experiment 2 were identical to those in Experiment 1 .

\section{Procedure}

The procedure was the same as in Experiment 1, except for a few changes. As before, the subjects viewed the digit display and then responded immediately to a probe if it appeared. They reported the single target digit when asked by the computer. Unlike in Experiment 1 , only an SOA of $150 \mathrm{msec}$ was used. The subjects were told that the digits were more likely to appear at the expected locations than at the unexpected locations. As in Experiment 1, there were 12 blocks of 64 trials, and trial types were randomly distributed across blocks. The subjects were allowed the same amount of practice and the same number of breaks as in Experiment 1.

\section{Results}

Performance on the primary task was very good for all subjects. Error rate averaged below $2 \%$, and the individual error rate for every subject was below $7 \%$.

The subjects were again very accurate in detecting probes. The mean error rate for the probe task was only $0.9 \%$. The subjects made just as many errors when the digits were at expected locations $(0.9 \%)$ as when they were at unexpected locations $(0.8 \%)$. Error rates were calculated separately for each combination of digit locations (expected vs. unexpected), probe quadrant, and probe location within quadrant (digit vs. blank). These means ranged from $0 \%$ to $1.6 \%$. As before, the error rates were so low that we did not analyze them.

Probe RTs were trimmed using the same procedure as in Experiment 1. Fewer than 4\% of RTs were removed in trimming. Figure 5 shows mean probe RTs for both the expected-location condition and the unexpected-location condition. As in Experiment 1, the data were first analyzed to show a basic attentional advantage for the target 


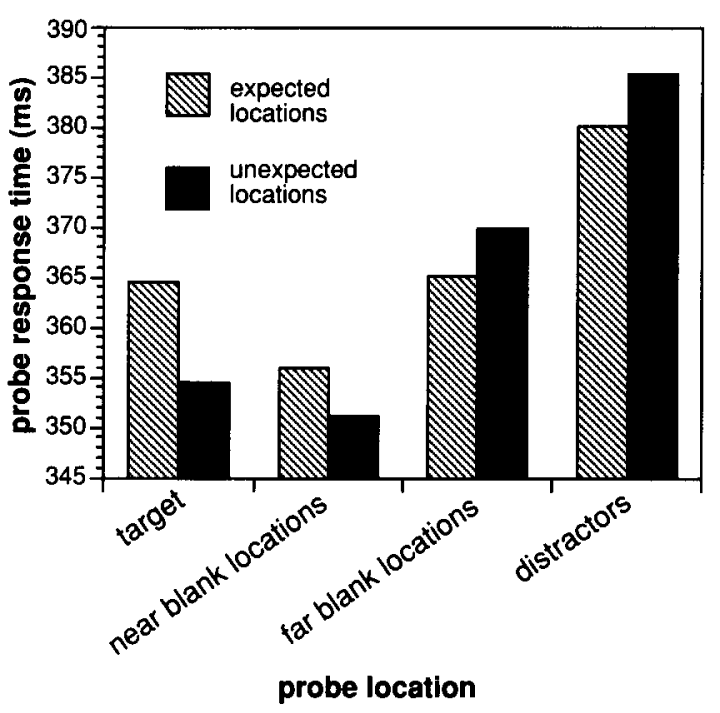

Figure 5. Probe response times for expected and unexpected conditions in Experiment 2.

over the distractor locations. A second set of analyses tested for a spread in attention to blank locations, and a third tested for attentional differences between digit and blank locations. Experiment 2 addressed a fourth question: Do subjects learn to expect stimuli at certain locations and allocate attention accordingly? The final set of analyses are devoted to this question.

\section{Digit Locations}

Probe RTs from only the digit locations were submitted to a repeated measures ANOVA, with probe location as the only within-subjects factor. Separate analyses were performed for expected- and unexpected-location conditions. The four possible probe locations were target, quadrant across the midline nearest the target, quadrant across the other midline from the target, and quadrant across both midlines from the target. For each ANOVA, a planned contrast compared the target location against the three distractor locations combined. The target location was significantly faster than the distractor locations for both the unexpectedlocation condition $[F(1,63)=15.16, p<.001]$ and the expected-location condition $[F(1,63)=7.98, p<.01]$.

As in Experiment 1, we tested for differences among the three distractor locations, which would be expected if the effects of attention decrease with distance from the target or if attention is allocated only to one hemisphere or quadrant. We employed a Tukey test to perform all three of the possible pairwise comparisons of the three distractor digit locations. As before, no difference in probe RT was found between any of the distractor locations in either the expected-location or the unexpected-location condition, suggesting that all distractor locations were treated equally by the attention mechanism.

\section{Blank Locations}

The second set of analyses tested for attentional differences between the blank locations. The analyses were based on a repeated measures ANOVA, with probe location as the only within-subjects factor, and the same contrasts were performed as in Experiment 1. Separate analyses were done for expected- and unexpected-location conditions.

One planned contrast tested whether the two blank locations near the target received more facilitation or inhibition than the two locations farther from the target. In Experiment 1, this contrast showed significantly faster responses near the target. The same pattern appeared in Experiment 2 for both the expected-location condition $[F(1,63)=4.52, p<.05]$ and the unexpected-location condition $[F(1,63)=5.23, p<.05]$. Facilitation at the target location and/or inhibition at distractor locations spills out somewhat to empty locations nearby.

As in Experiment 1, a second set of planned contrasts tested for midline effects between positions that were equally distant from the target. The first compared the two blank locations near the target: one that was in the same quadrant as the target, and one that was across either the horizontal or vertical midline. The other compared the two farther positions: one that was in the quadrant opposite the target and, thus, across both midlines, and one that was across only one midline from the target. Neither contrast was significant for either expected or unexpected locations ( $p \mathrm{~s}>.19$, in all four cases).

\section{Digit Versus Blank Locations}

The third set of analyses tested for differences between digit locations and blank locations. The first test determined whether the target location received different attentional treatment from the nearby blank areas. Repeated measures ANOVAs included three probe locations: target, neighboring blank location within target quadrant, and neighboring blank location across a midline from the target quadrant. In both expected- and unexpected-location conditions, planned contrasts showed that the RT for the target location was higher than the mean for the two neighboring locations, although the difference was not significant in either [for expected, $F(1,42)$ $=2.71, p>.10$; for unexpected, $F(1,42)<1.0]$. Target locations again seem to be treated similarly to blank locations, although there may be some hint of inhibition at the target location.

The second test compared the three distractor locations with the three blank locations in distractor quadrants. As before, probes at the distractor digit locations produced slower RTs than did probes at the blank locations for both the expected-location condition $[F(1,42)=18.09, p<$ $.001]$ and the unexpected-location condition $[F(1,42)=$ $10.37, p<.01]$. These slower responses could be attributed to inhibition of distractor locations, or to masking, as discussed below.

\section{Expected Versus Unexpected Locations}

Finally, two more ANOVAs compared probe RTs when the digits appeared at expected and unexpected locations. The first analysis included only data from digit locations. The factors were probe location (target, quadrant 
across the midline nearest the target, etc.) and expectedor unexpected-location condition. A planned contrast within this ANOVA tested whether the advantage for target over the three distractor locations described above varied between expected- and unexpected-location conditions. With expected locations, the target location RT was $364 \mathrm{msec}$, and the mean for the three distractor locations was $380 \mathrm{msec}$, producing a $16-\mathrm{msec}$ attentional advantage for the target. With unexpected locations, the target RT was $354 \mathrm{msec}$, and the three distractors averaged $385 \mathrm{msec}$, producing a $31-\mathrm{msec}$ attentional advantage. Although the target enjoyed a larger attentional advantage over the distractors in the unexpected-location condition than in the expected-location condition, the planned contrast indicated that the difference was not significant $[F(1,63)=2.56, p>.10]$. The overall interaction between probe location and expected/unexpected location, though, was significant $[F(3,63)=3.01, p<.05]$. Also, there was no main effect of expected versus unexpected locations $(F<1.0)$. The subjects' expectations for stimulus locations might have affected their attentional allocation somewhat, but any effects were fairly subtle. Perhaps larger effects could be produced by decreasing the percentage of unexpected-location trials or by giving the subjects more practice with the primary task.

A second ANOVA included only data from the four blank locations. The two factors were (1) probe location relative to target and (2) expected/unexpected location. There was no difference between expected- and unexpected-location conditions $(F<1.0)$ and no interaction between this factor and probe location $[F(3,63)=1.63$, $p>.15]$.

\section{Discussion}

Experiment 2, like Experiment 1, showed attentional selection favoring the target location over the distractor locations. Also, as in Experiment 1, selection seemed to take the form of inhibition of distractor locations relative to the background. The data show some hint of a difference in attentional allocation when the digits appear at unexpected locations, but the difference is quite small compared with the large differences between target and distractor locations. Although the subjects are focusing inhibition primarily on digit locations, they are not doing it by identifying those locations and inhibiting them in advance. Instead, the inhibition seems to be driven primarily by the presence of the stimuli, and not by expectations about stimulus location. A similar set of experiments by Kim and Cave (1995) measured allocation of spatial attention in conjunction search tasks (e.g., detecting a red square target among red circle, green square, and green circle distractors). In those searches, attention was allocated to favor the target location, but locations with either the target color or the target shape were also favored somewhat. The amount of attention at each location was determined by the target features present there, and not by its distance from the target. Taken together, all these experiments suggest that, in these discrimination tasks with distractors, the degree to which a particular location is se- lected depends mainly on the features present at that location. Of course, many cuing experiments have demonstrated that expectations for location can drive spatial attention when the target position is known in advance. However, when subjects do not have a cue to indicate which locations will have the target and which will have distractors, they apparently rely mainly on stimulus properties to drive attention. The control of attention more by stimulus properties than by expectation is what would be expected from a distributed attention mechanism. Such a result could also be produced by a centralized attention mechanism that did not rely on expectations; however, using expectations would be one of the main advantages of a centralized mechanism. In the General Discussion section, we will consider what type of distributed mechanism could implement this type of feature-driven selection.

Are there any conclusions to be drawn from the small expectation effect that does appear? A trend suggests that the attentional advantage for the target location over the distractor locations might be slightly stronger for the unexpected-location condition. Perhaps inhibition is allocated and deallocated to the distractor locations somewhat faster when the digits appear at their expected locations and when the probes appear as the deallocation is starting. Until further evidence is available, however, this explanation is just speculation. Only a single SOA was used here, and tests with other SOAs might reveal differences in the timing of attention between the expectedand unexpected-location conditions.

Another possible explanation for the expected/unexpected differences comes from an attentional model proposed by Houghton and Tipper (1994). In this model, the amount of distractor inhibition is adjusted according to the perceptual activation generated by the distractor. If the novelty of the unexpected distractor causes it to generate a higher activation, then it may receive more inhibition as well. Any such effect is small, however, indicating that the effect of expectation is much smaller than the effect of stimulus color.

As in Experiment 1, there is no hint of difference in attentional effects across vertical and horizontal midlines. Attention allocated to identify these digit targets and prevent interference from digit distractors does not seem to follow the patterns found by Hughes and Zimba (1985, 1987) and by Rizzolatti et al. (1987). Perhaps the more complex task in these experiments requires more careful control of attention across the visual field. If attention varied across midlines and across distances in these experiments as it did in the cuing experiments, it might have hampered the identification of the targets. In the cuing tasks, the pattern of attention across the visual field might not have been as important to successful completion of the task.

\section{EXPERIMENT 3 Spreading of Attention}

Cave's (1995) probe experiments indicated that attention allocated to stimulus locations might spread some- 
what. In Experiment 3, the probes were placed peripherally to the digit locations in order to measure the degree to which attentional effects spread. Moving the probes away from the digits also lessens the opportunities for the digits to mask the probes.

\begin{abstract}
Method
Subjects

Forty students at Vanderbilt University participated in this experiment, 20 attending to green digits, and 20 attending to red. All were fulfilling an introductory psychology course requirement. None knew the purpose of the experiment beforehand, and none had participated in the previous experiments. All the subjects reported normal or corrected-to-normal vision, including normal color perception. Most subjects finished in less than $1 \mathrm{~h}$.
\end{abstract}

\section{Apparatus}

This experiment was conducted with the same computers used in Experiments 1 and 2. The subjects responded via custom-built response keys that were connected to Strawberry Tree parallel interface cards. Responses were timed with clocks on the interface cards. This system provided more accurate response timing than the Macintosh keyboard. A chinrest was used to keep the subjects $58 \mathrm{~cm}$ from the computer display.

\section{Stimuli}

The digit arrays and probes were similar to those in Experiment 1 , except that each probe was located $15 \mathrm{~mm}\left(1.5^{\circ}\right.$ visual angle $)$ peripherally to its corresponding digit location. Thus, each probe appeared at one of eight locations equally spaced along an imaginary circle $75 \mathrm{~mm}$ ( $7.4^{\circ}$ visual angle) from fixation, as shown in Figure 6.

\section{Procedure}

The procedure was generally the same as in Experiment 1, with half the subjects attending to red and half attending to green. Instead of SOAs of 120,150 , and $180 \mathrm{msec}$, the SOAs in Experiment 3 were 90,120 , and $150 \mathrm{msec}$. The longest SOA was dropped to remove any chance of saccades. The probe display time was also cut from 60 to $30 \mathrm{msec}$, so that the entire interval from onset of the search display to offset of the probe was never more than $180 \mathrm{msec}$. As in Experiment 1, there were 12 blocks of 64 trials, and trial types were randomly distributed across blocks. The subjects were allowed the same amount of practice and the same number of breaks as in Experiment 1.

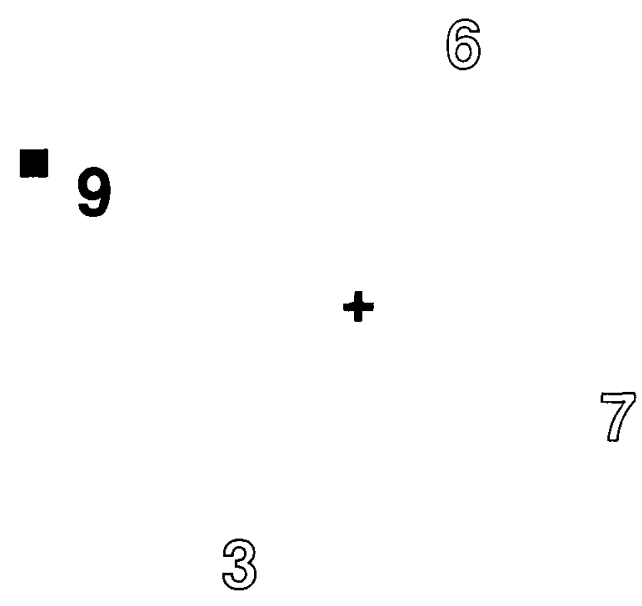

Figure 6. An example of the relative locations of the digit array and the probe in Experiment 3.

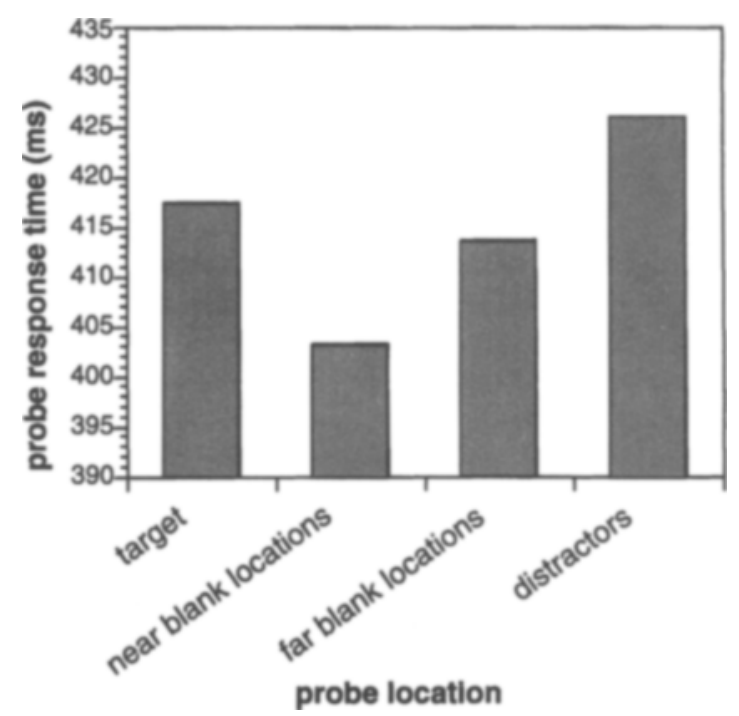

Figure 7. Probe response times for Experiment 3.

\section{Results}

Mean error rate for the primary task was $2 \%$, and all subjects averaged less than $6 \%$. The mean error rate for the probe task was $2 \%$. One subject produced $13 \%$ errors ( $4 \%$ false alarms and $22 \%$ misses), and the remaining subjects had probe error rates below $8 \%$. As before, trials with errors on the primary task were removed from the analyses, and the data were trimmed iteratively, removing less than $4 \%$ of the data. Figure 7 shows the probe RTs combined across the different combinations of SOA and target color. As in Experiment 1, SOA and color were included as factors in these analyses, but the few effects and interactions involving these factors did not seem to affect the overall conclusions, and they will not be reported.

\section{Digit Locations}

As in Experiments 1 and 2, probe RTs from only the digit locations were submitted to a repeated measures ANOVA, with probe position as a factor. Within each ANOVA, a planned contrast compared the target location against the three distractor locations combined. In the overall analysis, the target RT was only marginally faster than distractor RTs $[F(1,114)=3.53, p=.06]$. Although the effects were weak, attentional effects did seem to be spreading beyond the digit locations to regions farther in the periphery.

We employed a Tukey test to perform all three of the possible pairwise comparisons of the three distractor digit locations. No difference in probe RT was found between any of the distractor locations.

\section{Digit Versus Blank Locations}

RTs for probes next to the three distractor locations were compared with those next to the three blank locations in the same quadrants, using an ANOVA with both digit versus blank position and quadrant as factors. RTs 
were consistently slower for the distractor locations $[F(1,38)=29.26, p<.001]$.

A separate ANOVA included only R'Ts from probes next to the target location and the two blank locations on either side of it. A planned contrast within the overall analysis showed that the target probe yielded slower responses than did the blank probes $[F(1,76)=8.61, p<$ $.01]$. Experiments 1 and 2 also showed slower responses at the target location, although the difference was not significant. The larger RT difference in Experiment 3 might have been due to the faster SOA $(90 \mathrm{msec})$ used here. All digit locations might have been inhibited early on, with the inhibition lifting at the target location once it was determined.

\section{Blank Locations}

Another planned contrast compared probes next to the two blank locations on either side of the target, one within the target quadrant and one within a neighboring quadrant. The RTs were not significantly different $(F<1.0)$. An additional planned contrast showed no significant difference between the two blank locations far from the target $(F<1.0)$. As in Experiments 1 and 2, attentional allocation did not change across the quadrant boundary.

The two blank locations near the target produced faster RTs than did those far from the target $[F(1,114)=9.21$, $p<.01]$. As in Experiments 1 and 2, the inhibition from distractor locations seemed to spread to the regions between them.

\section{Discussion}

Probing locations peripheral to the stimulus locations yielded attentional effects similar to those found at the stimulus locations. The regions around distractor locations were inhibited relative to the region around the target location, and the distractor digit regions were very strongly inhibited relative to the blank areas between digits. As processing developed, the blank areas next to the target also began to show faster responses than did those far from the target. As before, attention effects were equal on both sides of the midline nearest the target.

In Experiments 1 and 2, target probe RTs were somewhat slower than RTs for the blank locations near the target; in Experiment 3, the target probe RTs were significantly slower. This effect may indicate that all digitstargets and distractors - mask the probes somewhat. Alternatively, the target location may receive some attentional inhibition at some time during the task. Perhaps, as suggested above, all stimulus locations are inhibited at first, and, then, when the target color is located, inhibition is removed from that location. This "inhibit-everything-first" might be used in this task because each digit location has a $75 \%$ probability of having a distractor.

Alternatively, the inhibition at the target location may occur after the target has been identified, and another element has been selected. This inhibition could be the "inhibition of return" demonstrated by Posner and Cohen (1984) and others in tasks in which attention is first al- located to a target location and then allocated somewhere else in response to a cue.

In Experiments 1 and 2, the slow responses at the distractor locations relative to the blank locations may be attributed either to attentional inhibition or to masking. In Experiment 3, masking was less likely, because the probe was positioned away from the digits; however, the difference between distractor and blank locations was still very strong. Experiment 4 provided a more complete control for masking.

\section{EXPERIMENT 4 Masking or Attentional Inhibition?}

In Experiments 1-3, the responses to probes at distractor locations were consistently much slower than to probes at blank locations between the distractors. These RTs suggest an inhibition that is applied in a fairly specific way to the distractor locations, but not to the blank regions. However, the distractor RTs may simply be slower because the digits mask the probe, making it more difficult to detect. Experiment 4 used a different stimulus designed to equalize masking of the probe across all locations. The entire display area was covered by a grid, and the four search elements were created by changing the color of some of the grid lines within a small region. As in Experiments $1-3$, the target element differed in color from the three distractor elements, and the subjects reported a property of the target (in this case, orientation). Whether the probe appeared at one of the element locations or at one of the "blank" locations between elements, it was surrounded by lines from the grid.

Experiment 4 also investigated the degree to which attention in this task was controlled by featural differences between the target and distractors (bottom-up factors) and the degree to which attention depends on subjects' knowing about the target and actively seeking it (topdown factors). The distinction between top-down and bottom-up attentional control is a part of many attention theories, including the Guided Search (Cave \& Wolfe, 1990; Wolfe, 1994) and FeatureGate (Cave, 1998) models. To allow these comparisons, Experiment 4 included three different conditions. The top-down condition was equivalent to that in Experiments 1-3: Probes measured spatial attention as subjects reported the orientation of a color singleton target. The bottom-up condition tested the degree to which attention was allocated to the singleton when subjects were not required to process it. The stimuli were the same as in the top-down condition, but subjects were given no primary task. They responded only to the probe. A separate control condition tested whether probes were masked more at the locations with search elements than at the blank, or background, locations. Its stimuli were very similar to the others, but there was no color singleton. The masking control and bottom-up attentional conditions were conducted in the first testing session, in that order; the top-down attentional condition was conducted in the second testing session. 


\section{Method}

\section{Subjects}

Forty students at the University of Illinois at Urbana-Champaign (mean age $=20.5$ years; range $=18-22$ years) participated in all three conditions. None knew the purpose of the experiment beforehand. All subjects had normal or corrected-to-normal vision using the Snellen acuity chart, and all performed adequately on the Ishihara test. The subjects performed two separate sessions. Most subjects finished in just over $1 \mathrm{~h}$ per session.

\section{Apparatus \\ This experiment was conducted using Intel-486-based comput- ers. The subjects responded using a standard PC keyboard. The sub- jects were seated $80 \mathrm{~cm}$ from the monitor; a chinrest was not used in this experiment.}

\section{Top-Down Attentional Condition}

Stimuli. During the entire experimental procedure, a grid made up of horizontal and vertical blue lines filled the display. The grid was composed of $1.5-\mathrm{mm}$ lines $\left(0.11^{\circ}\right.$ visual angle) centered $6.5 \mathrm{~mm}\left(0.47^{\circ}\right.$ visual angle $)$ apart. The primary stimulus had four display elements, centered on an imaginary circle $61 \mathrm{~mm}$ in diameter $\left(4.36^{\circ}\right.$ visual angle). Total screen size covered by the background grid was $27 \times 20 \mathrm{~cm}\left(18.6^{\circ} \times 14.0^{\circ}\right.$ visual angle $)$. Each stimulus was made by changing the color of a subset of the line segments in a region of the grid. Figure 8 shows the central part of the grid, including the locations of the search elements. As in Experiments $1-3$, all four elements were equally spaced along an imaginary circle around fixation, and each was positioned $22.5^{\circ}$ rotationally from one of the midlines. For half the subjects, the elements were offset clockwise from the midlines; for the other half, they were offset counterclockwise. The grid lines were blue, and the background was gray. Each element was composed of either horizontal or vertical line segments; for each element, the line segments were all either green or red. The shades of green, red, blue, and gray were matched for luminance using a Minolta Luminance Meter (C.I.E. relative photopic luminosity curve). Unlike in Experiments $1-3$, the matches were done separately for each of the four monitors used in the experiment. The luminances of the red, green, blue, and gray parts of the display were $3.6 \mathrm{~cd} / \mathrm{m}^{2}$ for all three monitors. For half the subjects, the singleton target was green, and the three distractors were red; for the other half, the colors were reversed. The orientation of the line segments was chosen randomly and independently for each element. The probe was very similar to the probe in Experiments 1-3. It was a black square, $4 \mathrm{~mm}$ on each side $\left(0.29^{\circ}\right.$ visual angle $)$, positioned in the center of one of the regions covered by the primary stimulus elements or at one of the four locations between two elements, and it always appeared after the primary stimulus elements had reverted to blue. A 5-mm black fixation spot $\left(0.36^{\circ}\right.$ visual angle $)$ was present throughout the experiment.

Procedure. The procedure in the top-down attentional condition was very similar to that in Experiments 1-3. At the beginning of each trial, the subjects fixated a black circle in the center of the display. The primary stimulus appeared for $98 \mathrm{msec}$. On the $50 \%$ of trials in which the probc appeared, it appeared 140,182 , or $224 \mathrm{msec}$ after the onset of the primary stimulus, and it remained visible for $42 \mathrm{msec}$. When the subjects detected the probe, they pressed the space bar as quickly and accurately as possible, and the RT was recorded. After a delay of $1,800 \mathrm{msec}$ from the probe offset time, the computer presented the word orientation in the center of the display, and the subjects responded by pressing one of two keys ( $F$ and $J$, counterbalanced) for horizontal or vertical. The intertrial interval was $1,700 \mathrm{msec}$. The subjects were instructed not to

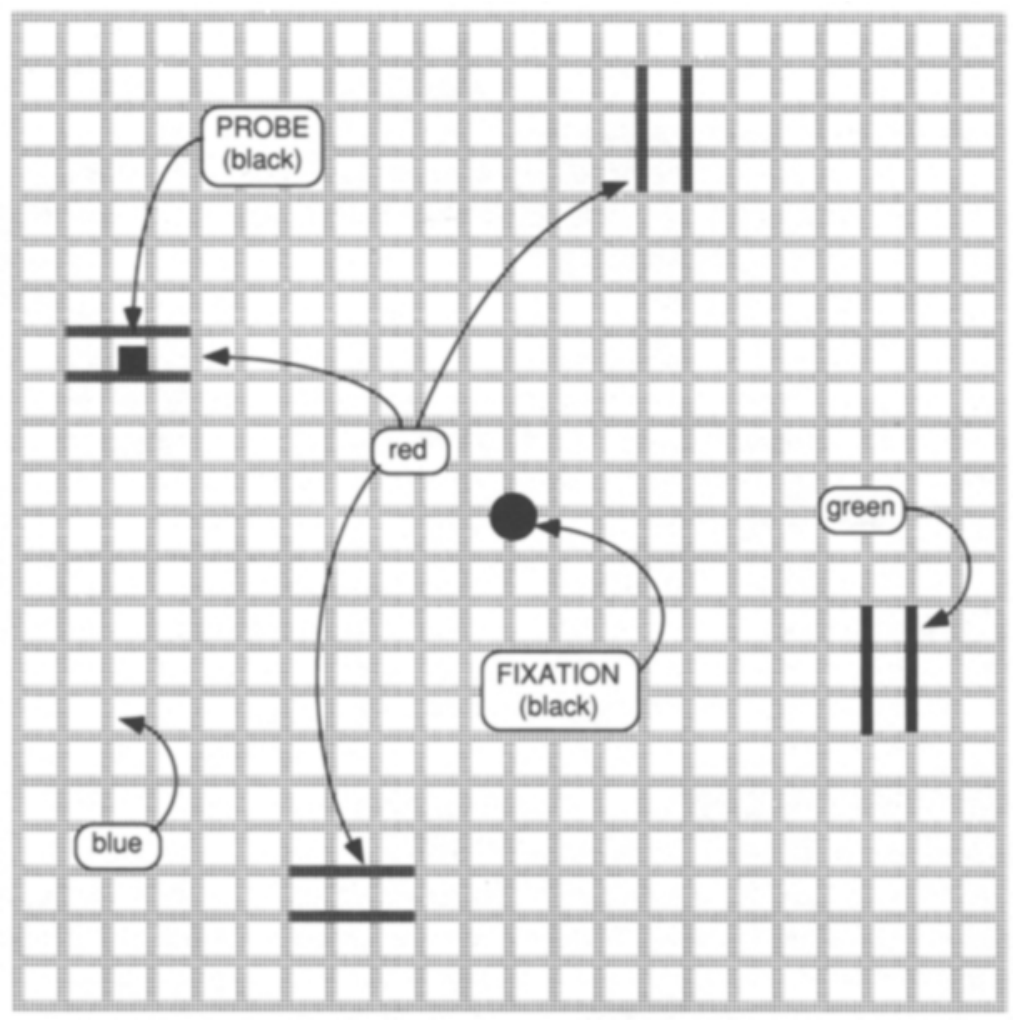

Figure 8. Arrangement of the primary stimulus for Experiment 4. One of the possible probe positions is also shown, in the center of the upper left search element. 
rush the orientation (primary task) response. A break occurred after every 64 trials. Each subject performed a total of 768 trials in this condition of Experiment 4.

\section{Bottom-Up Attentional Condition}

Except as noted below, the bottom-up attentional condition was conducted exactly like the top-down attentional condition.

Stimuli. The stimuli in the bottom-up attentional condition were the same as those in the top-down attentional condition.

Procedure. The bottom-up attentional condition included only the probe detection task. There was no search task. The subjects merely responded to the probes when they appeared by pressing the space bar. Because many trials required no response at all, the subjects initiated each trial by pressing a key ( $F$ or $J$, counterbalanced) when the word ready appeared in the center of the display. As before, each display contained either a red or a green color singleton. The subjects could take breaks whenever they desired. Each subject completed a total of 384 trials in this condition of Experiment 4. The bottom-up attentional condition was always conducted before the top-down attentional condition.

\section{Masking Control}

Except as noted below, the masking control condition was conducted exactly like the bottom-up attentional condition.

Stimuli. All four display elements in a single trial had the same color, so that no element would "pop out" from the rest and automatically draw attention (Theeuwes, 1991, 1992). The color of the elements varicd randomly from trial to trial, and each element's orientation was determined randomly and independently.

Procedure. For the masking control, the subjects performed only the probe detection task. Each subject completed a total of 384 trials in the control condition of Experiment 4.

\section{Results}

\section{Masking Control}

The probe RTs from the control experiment showed no evidence that display elements masked the probes. RTs for locations formerly occupied by a display element were just as fast as RTs for the blank locations between display elements, as shown in Figure 9.

As in Experiments 1-3, a trimming procedure iteratively removed the highest and/or lowest RTs in a condition if they were more than $3.5 \mathrm{SD}$ s from the mean of the remaining RTs. This trimming removed $3.5 \%$ of the probe RTs.

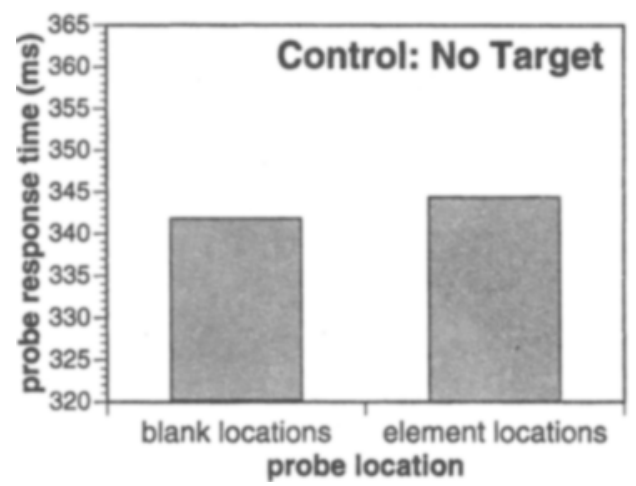

Figure 9. Probe response times for the control condition of Experiment 4.
The subjects made errors at probe detection on only $0.4 \%$ of the trials $(0.3 \%$ misses and $0.4 \%$ false alarms $)$. A repeated measures ANOVA, with probe location (element location or blank location) and SOA as withinsubjects factors, showed no significant effect of probe location $(F<1)$. Probe RT was significantly increased at longer SOAs $[F(2,78)=17.4, p<.0001]$. There was no interaction between SOA and probe location $(F<1)$.

\section{Bottom-Up Attentional Condition}

The subjects made errors at probe detection on only $0.7 \%$ of the trials $(0.5 \%$ misses and $0.8 \%$ false alarms). Trimming removed $2.7 \%$ of the probe RTs. The remaining RTs, shown in Figure 10, were submitted to a repeated measures ANOVA, with probe location and SOA as within-subjects factors and singleton color as a betweensubjects factor. The analysis showed significant effects of probe location $[F(7,266)=3.0, p<.01]$, which were explored with contrasts presented below. Probe RT was significantly increased at longer SOAs $[F(2,76)=31.5$, $p<.0001]$. Probes were detected more quickly when a red singleton was present than when a green singleton was present $[F(1,38)=6.9, p<.05]$. No significant interactions were present (all $p \mathrm{~s}>.07$ ).

\section{Top-Down Attentional Condition}

The subjects made errors at probe detection on only $0.3 \%$ of the trials $(0.4 \%$ misses and $0.2 \%$ false alarms). Mean error rate at the primary task was $3.7 \%$ (highest error rate for any single subject was $16 \%$ ). Probe responses from trials with an error on the primary task were excluded from the analysis. As before, a trimming procedure iteratively removed the highest and/or lowest RTs in a condition if they were more than $3.5 S D$ s from the mean of the remaining RTs. The trimming removed $1.5 \%$ of the probe RTs. Figure 11 shows the probe RTs, which were submitted to a repeated measures ANOVA, with probe location and SOA as within-subjects factors and singleton target color as a between-subjects factor. It showed significant effects of probe location $[F(7,266)=6.0, p<$ $.0001]$, which were explored with contrasts described below. As in the bottom-up condition, probe RT was significantly increased at longer $\operatorname{SOAs}[F(2,76)=25.5, p<$ $.0001]$, and probes were detected more quickly when a red singleton was present than when a green singleton was present $[F(1,38)=4.1, p<.05]$. No significant interactions were present (all $p s>.07$ ).

\section{Comparisons of Attentional Effects at Different Locations}

As in Experiments 1-3, attentional effects at different locations in the display were compared with planned contrasts. The contrasts were conducted separately for the bottom-up and top-down conditions. In general, both conditions showed similar patterns, but the effects were weaker and sometimes not significant in the bottom-up condition. 


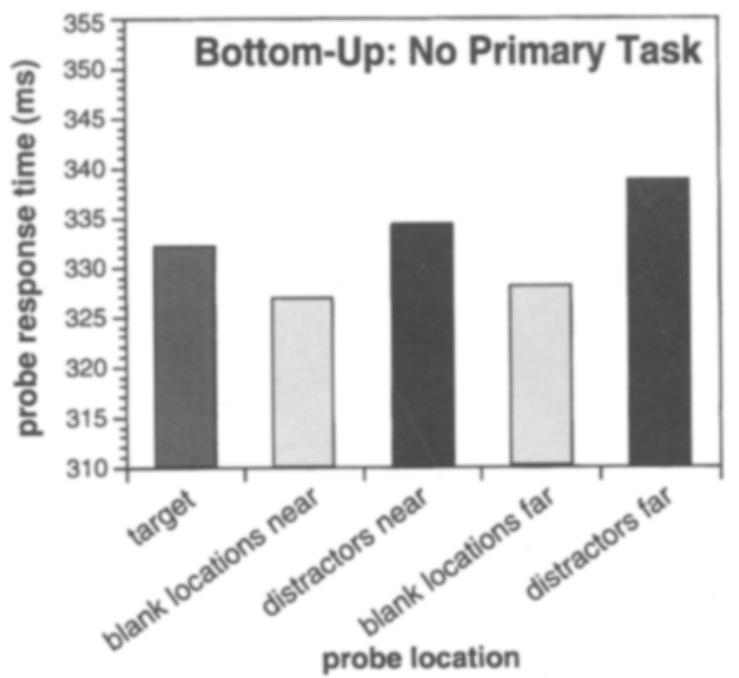

Figure 10. Probe response times for the bottom-up attention condition of Experiment 4.

Singleton target versus distractor locations. RT for the target (color singleton) location was significantly faster than that for the distractor locations in the topdown attention condition $[F(1,266)=5.0, p<.05]$. This is evidence that spatial selection was used when the color singleton was relevant to the task. The color singleton location also produced faster responses than did the distractor locations in the bottom-up condition, but the difference was not significant $[F(1,266)=1.3, p=.25]$.

Element versus blank locations. Probes at blank locations elicited faster responses than did probes at element locations in the same quadrants for both bottom-up and top-down attentional conditions $[F(1,266)=11.9$, $p<.001$, and $F(1,266)=28.0, p<.0001$, respectively].

Also, the target RTs were significantly slower than the two blank location RTs on either side of the target in the top-down condition $[F(1,266)=5.3, p<.05]$. This might reflect inhibition applied before the target color was detected, or it might be inhibition of return applied after the target processing was finished. The bottom-up condition showed the same pattern, but the difference was not significant $[F(1,266)=2.5, p=.11]$. The results of the control condition indicate that, in this case, the slower target RTs were not due to masking.

Distance from singleton location. In both conditions, probes at the two blank locations near the singleton produced faster responses than did those at the two blank locations farther from the singleton; however, in neither case was the difference significant $(p s>.20$ ).

Midline effects. Planned contrasts showed no differences between RTs for the blank location within the quadrant with the singleton and the blank location the same distance away from the singleton in the neighboring quad$\operatorname{rant}(F \mathrm{~s}<1.0)$ or between RTs for the two blank locations far from the singleton $(p s>.18)$.
Bottom-up versus top-down attentional effects. The difference in probe RT between bottom-up and top-down conditions, after equating target RT between the two conditions, is shown in Figure 12. The difference between probe RTs to the target relative to the blank locations near the target became greater when the color singleton target became relevant to the task. Additionally, the distractor locations near the target became more inhibited when the color singleton target was task relevant.

\section{Discussion}

Regardless of the target color, responses were slower for the distractor locations than for the blank locations in between them. Unlike in Experiments 1-3, these differences were not attributable to masking. In the control condition, responses to probes at stimulus locations and blank locations did not differ, demonstrating that the probes at the element locations were not masked any more than those at the blank locations in these displays. Thus, the effects in the attention conditions must reflect differences in attention. In order to identify the target, the subjects apparently inhibited the distractor locations, but the inhibition did not apply (or applied more weakly) to the blank locations between the distractors. When attention was driven only by stimulus salience, and not by the task demands, the attentional effects were weak or nonexistent. When attention was necessary for the task, however, its effects were quite strong.

These results suggest that the data from Wolfe and Pokorny (1990) and from Klein and Taylor (1994) might be reconsidered. Because they found slow responses for distractor location probes in both feature and conjunction searches, they attributed the results to masking rather than attention. Experiment 4 (along with its control condition) demonstrated one situation in which slow re-

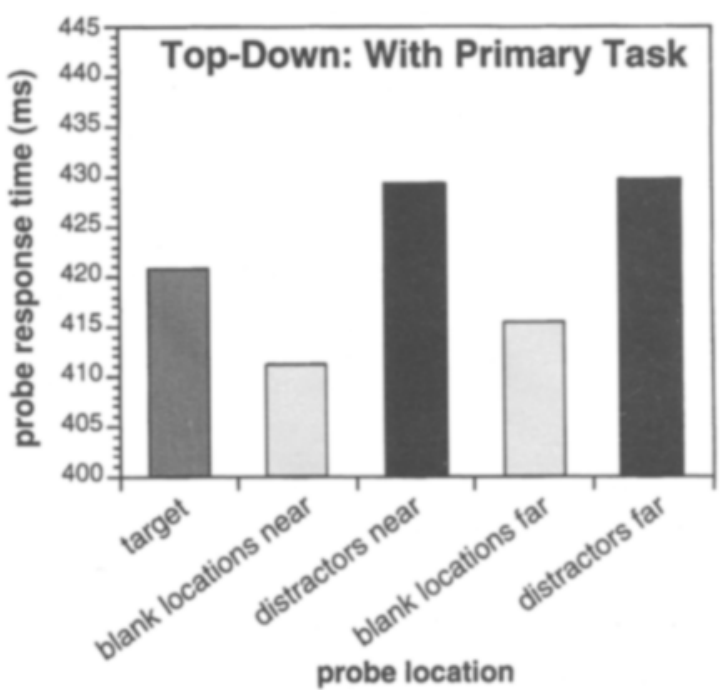

Figure 11. Probe response times for the top-down attention condition of Experiment 4. 


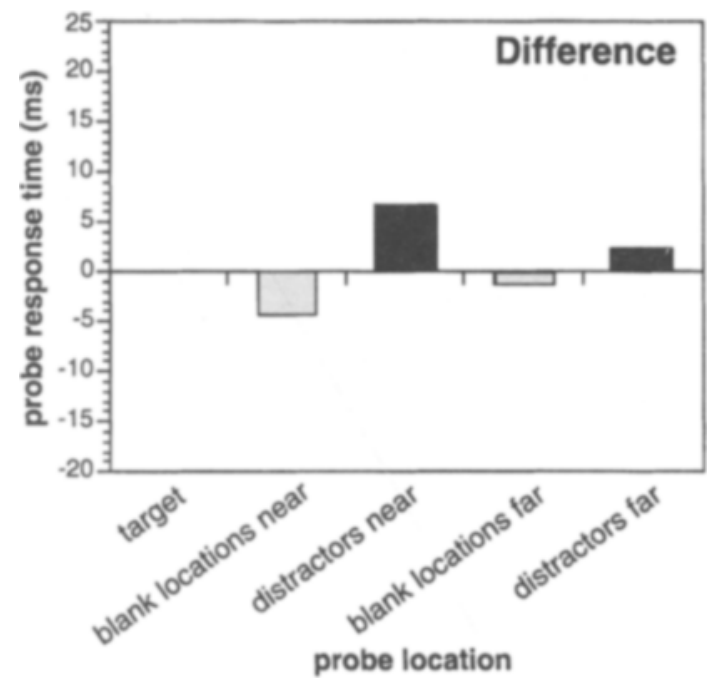

Figure 12. Top-down minus bottom-up probe response times, adjusted to equalize target location response times.

sponses for distractor locations could be attributed to attentional inhibition rather than masking. Kim and Cave (1995) also demonstrated that spatial selection is used in some feature searches as well as conjunction searches, raising the possibility that at least a portion of the effects measured by Wolfe and Pokorny and by Klein and Taylor was due to attentional inhibition of distractors.

Because of the difficulty of locating the targets in this task, the SOAs were longer in Experiment 4 than in Experiments $1-3$, raising the possibility of saccades in the two longest SOAs, even though the subjects were instructed not to saccade during trials. The faster responses for target over distractor locations were probably not due to saccades to the target location, because no interaction involving SOA was present. More importantly, saccades to the target location would not produce slower RTs at the distractor locations than at the blank locations.

\section{GENERAL DISCUSSION}

The pattern of RTs in these experiments shows that target locations are selected over distractor locations in these tasks in a way that affects the processing of other stimuli appearing subsequently at these locations. These results are consistent with other probe RT studies by Banks et al. (1992), Cave (1995), Cave and Zimmerman (1997), Kim and Cave (1995), and Kramer, Weber, and Watson (1995). They are also consistent with cuing experiments by Folk, Remington, and Johnston (1992), which showed that a color singleton cue can draw attention to its location, and with experiments by Theeuwes (1991, 1992, 1994, 1995, 1996), which showed that a color singleton that is irrelevant to a task can draw attention away from a target during search.

The present experiments also showed a very strong attentional difference between distractor locations and the blank regions between the digits. In this task, target information is apparently selected (separated from potentially interfering distractor information) by inhibiting those locations that contain distractor features. Horowitz (1995) found similar results by measuring how the RT on each trial in a search task varied depending on the location of the target in previous trials.

These results are hard to reconcile with spotlight (Posner et al., 1980) and zoom lens (Eriksen \& St. James, 1986; Eriksen \& Yeh, 1985) metaphors of spatial attention, all of which posit a single area of facilitation. These metaphors would predict that the inhibition of nontarget locations relative to target locations should be uniform or should increase with distance from the target. Spatial attention may be more like a spotlight, zoom lens, or gradient of facilitation when it is allocated in response to spatial cues, especially endogenous cues that require some interpretation (Jonides, 1981). Consistent with this is the finding by Folk and Remington (1996) that subjects were unable to allocate inhibition in response to an endogenous cue in the same way that they allocate facilitation. Also, distractor inhibition may become more important when there are more distractors to interfere. Sagi and Julesz (1986) used a probe procedure similar to the one described above to demonstrate a gradient pattern of facilitation around a target with only a single distant distractor present. However, when the target is accompanied by many distractors (Bahcall \& Kowler, 1995; Kröse \& Julesz, 1989), the nearby distractors do not benefit from a facilitation gradient, but they are strongly inhibited relative to the target. Data from Zimba and Hughes (1987) also suggest that distractor locations might be inhibited in a cuing task. All these results taken together suggest that when spatial attention is used in the course of identifying a target designated by a feature among distractors with a potential for interference, as in the experiments presented here, it seems to selectively inhibit those locations with stimuli that will interfere with the target, without inhibiting other locations. The model described below by Cave, Kim, Bichot, and Sobel (1998; Cave, 1998) attempts to explain these different patterns of attention.

Spatial attention in this task differs in another important respect from attention allocated in response to a spatial cue. In cuing experiments, attention drops off considerably between cued and uncued quadrants. However, in the present experiments, no attentional difference between quadrants was detected. Further tests are necessary to determine exactly when spatial attention is affected by quadrant boundaries. Perhaps activation or inhibition of an entire quadrant is easier than selection of a small region within a quadrant. When few distractors are present, subjects may merely activate the cued quadrant and/or inhibit the uncued quadrants; however, when distractors pose a threat of interference, subjects may expend extra effort to select target locations more narrowly. Interestingly, though, selection of an entire quadrant would have been sufficient to select the target over all distractors in these tasks, because only one element appeared in each 
quadrant. Another possibility is that the specification of locations to be selected by top-down mechanisms may not be very specific and may rely on separate mechanisms for each quadrant, whereas selection driven by stimulus features such as color may be spatially more specific.

Other researchers have offered alternatives to the spotlight metaphor. For instance, Sperling and Weichselgartner (1995) concluded that when attention shifts from one location to another, it does not slide across the visual field in an analog fashion. Like Yantis (1988) and Eriksen and Murphy (1987), they question the findings by Shulman, Remington, and McLean (1979) and by Tsal (1983) that were taken to demonstrate analog attentional movement, and they perform their own experiment showing that the time to move attention from a peripheral location to the fovea varies little with distance. However, they still maintain one aspect of the spotlight metaphor by assuming that attention takes the form of a single focused area of facilitation. Their data could also be explained by assuming that attention takes the form of inhibition focused on distractor locations. Cheal, Lyon, and Gottlob (1994) proposed their own alternative to the spotlight, in which a gradient filter controls the flow of information from different locations. Their gradient filter shares many aspects with the system we are proposing, although it relies on facilitation of target locations rather than inhibition of distractor locations.

Although selection is spatially more focused with the search task presented here than with some earlier cuing tasks, it is somewhat distributed. The attentional effects are still measurable at locations peripheral to the stimulus elements. Also, the attentional effects spread somewhat with time to the locations between the stimulus elements, producing slower RTs at the blank locations far from the target than at those close to the target at the longer SOAs. The pattern of spatial attention may be more spatially restricted for displays and tasks that are more complex than those used here. Unlike most natural scenes, these displays have very few distractors, and distractors and target were all spaced far apart. Further experiments using this probe technique can give a more complete map of the allocation of attention and can determine whether it becomes more focused when distractors are more numerous and within the same quadrant as the target.

The attentional strategy revealed by these experiments might be described as "blocking when necessary." Locations with interfering distractors are inhibited, but other locations are not. This strategy may be preferable to blocking large regions of the visual field unnecessarily. Regions that are blank at one moment may suddenly be filled with a stimulus that needs to be detected and identified quickly. A blocking-when-necessary strategy will not impede the processing of these stimuli. This strategy is similar to one proposed by Worden, Schneider, and Wellington (1995) and is consistent with the principle described by Lavie and Tsal (1994) that the allocation of spatial attention varies as necessary according to the per- ceptual load and also with the demonstration by Lavie (1995) that the influence of a distractor on response is weakened as perceptual load increases.

LaBerge (1995) has proposed a model that attempts to account for both behavioral and physiological evidence with separate mechanisms for inhibiting distractors and facilitating targets. His distractor inhibition comes from oculomotor mechanisms, and it can only be used when stimuli are far enough apart to trigger separate eye movements, as they were in the displays used here. In his model, target enhancement is used when two or more display elements are close enough that they are considered a single unit (LaBerge, 1995, pp. 120-121). Future experiments using spatial probes will test this claim by measuring whether distractor inhibition disappears and target facilitation appears when distractors are placed closer to the target. Although the distractor inhibition found here seems generally consistent with LaBerge's oculomotor inhibition, it apparently conflicts with his suggestion that attention to an object's location produces inhibition and attention to an object's features produces facilitation. In the experiments reported here, subjects reported shape or orientation, and location was irrelevant to the task, yet distractors were inhibited.

A pattern of distractor blocking also appears in many experiments demonstrating negative priming, in which the response to a stimulus is slowed when it appears at the same location as a distractor from an earlier display (Shapiro \& Loughlin, 1993; Tipper, Brehaut, \& Driver, 1990). This location-based negative priming might be related to the location-based inhibition that produces RT patterns in the probe experiments presented here. For example, Tipper et al. (1990) asked subjects to report the location of a target symbol that appeared along with a distractor symbol. This prime trial was immediately followed by a probe trial with a new target symbol and distractor symbol. Subjects were slower when the probe target appeared in the location previously occupied by the distractor on the prime trial than when it appeared in a previously unoccupied location. Rather than explaining these results on the basis of location-based inhibition, Tipper et al. (1990) proposed that two symbols appearing successively at the same location are perceived as a single object and that this negative priming results from an objectbased inhibition of an internal representation of the distractor object in the prime task. Contrary to the negativepriming paradigm, the present experiments used a neutral stimulus, a small dot, in the probe trial. Since the probe dot varied dramatically from the digits in size, color, and shape, it was less likely to be seen as a continuation of the same object. Thus, the slower probe RTs at distractor locations in the present experiments support the idea that the inhibition is location-based and support Shapiro and Loughlin's (1993) conclusion that location alone can explain the inhibitory effects found in many negativepriming experiments with static objects.

Park and Kanwisher (1994) offered an alternative explanation for Tipper et al.'s (1990) negative priming. They 
claimed that slower responses for targets at a distractor's location was caused not by inhibition of distractors but by the mismatch between the objects appearing at that location at different times. If the drastic difference between target digits and probes in color, size, and shape in these experiments prevents them from being perceived as the same object, then such a mismatch should not affect RTs here. Also, experiments by Tipper, Weaver, and Milliken (1995; see also Milliken, Tipper, \& Weaver, 1994) showed no evidence of slower responses due to mismatching stimuli when subjects consistently selected the target by the same property across trials, as the subjects did in the presents experiments.

Other experiments have demonstrated negative priming that is tied to a particular shape, regardless of its location (Tipper \& Cranston, 1985). This shape-based inhibition can last for a very long time (DeSchepper \& Treisman, 1996), suggesting that it results from a mechanism separate from that of the location-based inhibition found in the probe experiments. Ruthruff and Miller (1995) found that this shape-based negative priming did not occur when target and distractor locations were fixed throughout a block of trials. That is, when one location was always used for a target and another for a distractor, responses to the probe target that appeared in the previous primary trial as a distractor were not slowed. Apparently, when distractor inhibition can easily be controlled by inhibition of their locations at an early processing stage, the shape-based inhibition is unnecessary in the later stages.

Physiological studies have uncovered neural correlates to the inhibition of distractors in area V4 and sometimes in areas V2 and V1. Moran and Desimone (1985) and Luck, Chelazzi, Hillyard, and Desimone (1997) reported that spatially restricted focal attention suppressed a cell's response to a distractor when both target and distractor were located within the cell's receptive field. Motter (1993, 1994) found that responses to distractors were suppressed in visual searches with the target indicated either by a location cue or by color. Chelazzi, Miller, Duncan, and Desimone (1993) also found suppression of cells responding to distractors in search. Recently, Schall and colleagues, using search displays with a red singleton target among green distractors similar to those in these experiments, have found correlates of attentional modulation in responses of neurons in the frontal eye field (FEF). They found that the initial visual response of FEF visuomovement neurons is the same whether the singleton target or distractors fall in their receptive field, but the activity of these neurons evolves to discriminate the singleton as reflected by an attenuation of responses to distractors (Schall, Hanes, Thompson, \& King, 1995; Thompson, Hanes, Bichot, \& Schall, 1996; but see Bichot, Schall, \& Thompson, 1996). Furthermore, this modulation was not dependent on saccade production; rather, it reflected the outcome of an automatic process by which salient stimuli are detected (Thompson, Bichot, \& Schall, 1997).

Further evidence on the physiological mechanisms of attention comes from ERP studies. Luck and Hillyard (1994a, 1994b) demonstrated an ERP component, which they labeled N2pc, that was elicited by targets in the presence of distractors and by difficult nontargets in the presence of distractors, but not with isolated targets or easy nontargets. From this evidence, they concluded that the $\mathrm{N} 2 \mathrm{pc}$ component is involved in inhibiting or suppressing distractor items in order to allow the target to be selected. All these experiments provide useful clues as to how distractor inhibition might be implemented.

Selectively blocking distractor locations would require a more complex mechanism than an attentional spotlight if it were centrally controlled, because all of the distractor locations would have to be identified and tracked. "Blocking when necessary" could be implemented more easily, however, by a distributed mechanism with a selection gate at each visual field location. The information flowing through each gate is impeded according to both bottom-up and top-down factors (Cave \& Wolfe, 1990). The bottom-up mechanism will close the gates at locations with features that are very similar to the features at surrounding locations. The top-down mechanism will close gates at locations with one or more features that are inconsistent with the target. Once each local selector receives a top-down signal specifying target and distractor features, it can quickly inhibit its location if a distractor appears there. For the primary task in the experiments reported here, in which the target is both a color singleton and is known in advance, either the bottom-up or top-down mechanism alone would be sufficient to inhibit the distractors. Figure 13 shows how this feature-driven spatial selection would work in these

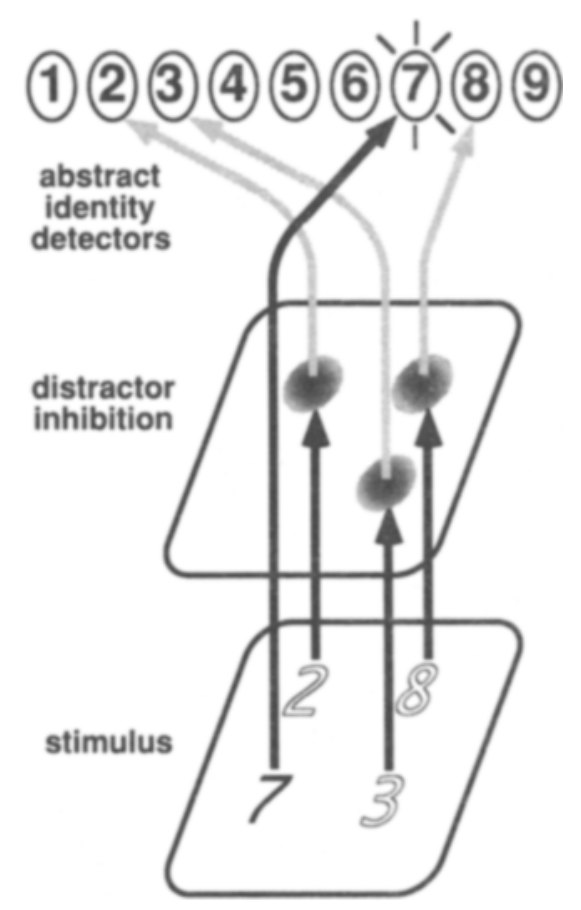

Figure 13. A simple mechanism for feature-driven inhibition of distractor locations. 
tasks. The distributed nature of this mechanism also explains how it can respond so quickly, allowing it to affect detection of probes presented very soon after the stimulus onset (just $30 \mathrm{msec}$ in Kim \& Cave, 1995). The fact that expectation of digit locations had such a small effect in Experiment 2 is also consistent with a distributed, feature-driven system.

Cave et al., $(1996,1998)$ have produced a detailed model showing how this fast feature-driven selection can be simply implemented in a neural network. The model is similar in many ways to a neural network model proposed by Houghton and Tipper (1994) to explain negative priming. In both models, targets are selected at least in part by inhibiting display elements that possess properties that do not match properties of the target. The models differ in that, in the Cave et al. model, locations are inhibited, whereas, in Houghton and Tipper's model, abstract object representations are inhibited. Cave (1998) explains how this model, which produces distractor inhibition, can also produce attentional gradients in response to endogenous cues, as discussed above.

The Cave et al. $(1996,1998)$ model is generally consistent with the physiological findings of Moran and Desimone (1985) and of Motter (1993, 1994), and it can serve as an implementation of Guided Search (Cave \& Wolfe, 1990; Wolfe, 1994) and other search models that rely on spatial selection driven by stimulus features (Treisman, 1988; Treisman \& Sato, 1990). Guided Search specifies what locations should be selected or inhibited, but it does not address how that inhibition is implemented or how blank regions of the visual field are treated. The Cave et al., model differs from Guided Search in that it can allow two noncontiguous locations to be selected simultaneously. Different studies disagree as to whether split attention is a desirable property in a model (Bichot, Cave, \& Pashler, in press; Castiello \& Umiltà, 1992; Heinze et al., 1994; Kramer \& Hahn, 1995; Neath \& Tarr, 1993; Posner et al., 1980). Experiments that do demonstrate a split of attention across regions may be the result of inhibition of distractor locations, rather than selection by multiple spotlights.

The nature of spatial selection in this task also has implications for object recognition. Some models of visual processing include an attentional spotlight or window that serves multiple functions. In addition to eliminating interference from distractor objects, it also produces a spatial representation of only the region of the visual field occupied by the selected object, scaled to a standard size (Kosslyn \& Koenig, 1992; Olshausen, Anderson, \& Van Essen, 1993). In other words, the attentional spotlight also provides position and size constancy. This sort of attention window is appealing because it simplifies the job of the recognition mechanism receiving its output. However, the pattern of RTs from the present experiments is less consistent with this type of attention window than with distractor inhibition. If visual selection is accomplished completely by inhibiting distractor locations, then the recognition system must be able to recognize an object that is still embedded in the background. Mozer's (1991) model shows how object recognition might be done without first using an attention window to produce a location-normalized representation. Alternatively, the task of selection may be split between an early, broadly focused mechanism that inhibits each distractor's general region and a later attention window that specifically selects only the target and normalizes it. Ultimately, understanding object recognition and the rest of higher level cognition will depend in part on studies such as these that reveal the nature of visual selection.

\section{REFERENCES}

BahCALl, D. O., \& Kowler, E. (1995). Attentional interference at close spatial separations. Investigative Ophthalmology \& Visual Science, 36(Suppl.), S901.

Banks, W. P., Kouwabunpat, D. T., \& Ciranni, M. A. (November, 1992). Patterns of activation in attended and unattended objects. Poster presented at the annual meeting of the Psychonomic Society, St. Louis, MO.

Bichot, N. P., CAVE, K. R., \& PASHLER, H. (in press). Visual selection mediated by location: Feature-based selection of noncontiguous locations. Perception \& Psychophysics.

Bichot, N. P., Schall, J. D., \& Thompson, K. G. (1996). Visual feature selectivity in frontal eye fields induced by experience in mature macaques. Nature, 381, 697-699.

Castiello, U., \& Umiltà, C. (1992). Splitting focal attention. Journal of Experimental Psychology: Human Perception \& Performance, 18, 837-848.

CAVE, K. R. (1995). Measuring the allocation of spatial attention. Unpublished manuscript.

CAVE, K. R. (1998). The Feature Gate model of visual selection. Manuscript submitted for publication.

CAVE, K. R., \& BICHOT, N. P. (in press). Visuo-spatial attention: Beyond a spotlight model. Psychonomic Bulletin \& Review.

CAVE, K. R., KIM, M.-S., BiChOT, N. P., \& SobeL, K. V. (1996, April). Modeling feature and conjunction searches with feature-driven selection. Poster presented at the third annual meeting of the Cognitive Neuroscience Society, San Francisco.

CAVE, K. R., Kim, M.-S., BICHOT, N. P., \& Sobel, K. V. (1998). Visual selection within a hierarchical network: The Feature Gate model. Manuscript submitted for publication.

CAVE, K. R., \& PASHLER, H. (1995). Visual selection mediated by location: Selecting successive visual objects. Perception \& Psychophysics, 57, 421-432.

CAve, K. R., \& Wolfe, J. M. (1990). Modeling the role of parallel processing in visual search. Cognitive Psychology, 22, 225-271.

CAVE, K. R., \& Zimmerman, J. M. (1997). Flexibility in spatial attention before and after practice. Psychological Science, 8, 399-403.

ChEAL, M. L., LyON, D. R., \& GotTLOB, L. R. (1994). A framework for understanding the allocation of attention in location-precued discrimination. Quarterly Journal of Experimental Psychology, 47A, 699-739.

Chelazzi, L., Miller, E. K., Duncan, J., \& Desimone, R. (1993). A neural basis for visual search in inferior temporal cortex. Nature, 363 , 345-347.

DeSchepper, B., \& Treisman, A. (1996). Visual memory for novel shapes: Implicit coding without attention. Journal of Experimental Psychology: Learning, Memory, \& Cognition, 22, $27-47$.

Downing, C. J., \& PINKER, S. (1985). The spatial structure of visual attention. In M. I. Posner \& O. S. M. Marin (Eds.), Attention and performance XI: Mechanisms of attention (pp. 171-187). Hillsdale, NJ: Erlbaum

ERIKSEN, C. W., \& Hoffman, J. E. (1974). Selective attention: Noise suppression or signal enhancement? Bulletin of the Psychonomic Society, 4, 587-589.

ERIXSEN, C. W., \& MURPhy, T. D. (1987). Movement of attentional 
focus across the visual field: A critical look at the evidence. Perception \& Psychophysics, 42, 299-305.

ERIKSEN, C. W., \& ST. JAMES, J. D. (1986). Visual attention within and around the field of focal attention: A zoom lens model. Perception \& Psychophysics, 40, 225-240.

ERIKSEN, C. W., \& YeH, Y.-Y. (1985). Allocation of attention in the visual field. Journal of Experimental Psychology: Human Perception \& Performance, 11, 583-597.

Folk, C. L., \& Remington, R. W. (1996). When knowledge does not help: Limitations on the flexibility of attentional control. In A. F. Kramer, M. G. H. Coles, \& G. D. Logan (Eds.), Converging operations in the study of visual selective attention (pp. 271-295). Washington, DC: American Psychological Association.

Folk, C. L., Remington, R. W., \& Johnston, J. C. (1992). Involuntary covert orienting is contingent on attentional control settings. Journal of Experimental Psychology: Human Perception \& Performance, 18, 1030-1044.

Heinze, H.-J., Luck, S. J., Münte, T. F., Gös, A., MANGun, G. R., \& HILlyARD, S. A. (1994). Attention to adjacent and separate positions in space: An electrophysiological analysis. Perception \& Psychophysics, 56, 42-52.

Hoffman, J. E., \& Nelson, B. (1981). Spatial selectivity in visual search. Perception \& Psychophysics, 30, 283-290.

Hoffman, J. E., Nelson, B., \& Houck, M. R. (1983). The role of attentional resources in automatic detection. Cognitive Psychology, 15, $379-410$.

Horowitz, T. S. (1995). Distractor inhibition in a visual search task. Investigative Ophthalmology \& Visual Science, 36 (Suppl.), S899.

Houghton, G., \& Tipper, S. P. (1994). A model of inhibitory mechanisms in selective attention. In D. Dagenbach \& T. H. Carr (Eds.), Inhibitory processes in attention, memory, and language (pp. 53-112). San Diego: Academic Press.

Hughes, H. C., \& ZimBA, L. D. (1985). Spatial maps of directed visual attention. Journal of Experimental Psychology: Human Perception and Performance, 11, 409-430.

Hughes, H. C., \& Zimba, L. D. (1987). Natural boundaries for the spatial spread of directed visual attention. Neuropsychologia, 25, 5-18.

JONIDES, J. (1981). Voluntary versus automatic control over the mind's eye's movement. In J. Long \& A. Baddeley (Eds.), Attention and performance IX (pp. 187-203). Hillsdale, NJ: Erlbaum.

KIM, M.-S., \& CAVE, K. R. (1995). Spatial attention in visual search for features and feature conjunctions. Psychological Science, 6, 376-380.

KLEIN, R. [M.] (1988). Inhibitory tagging system facilitates visual search. Nature, 334, 430-431

KleIN, R. M., \& TAYLOR, T. L. (1994). Categories of cognitive inhibition with reference to attention. In D. Dagenbach \& T. H. Carr (Eds.), Inhibitory processes in attention, memory, and language (pp. 53-112). San Diego: Academic Press.

KossLyn, S. M., \& KoeniG, O. (1992). Wet mind: The new cognitive neuroscience. New York: Free Press.

Kramer, A. F., \& Hahn, S. (1995). Splitting the beam: Distribution of attention over noncontiguous regions of the visual field. Psychological Science, 6, 381-386.

Kramer, A. F., Weber, T. A., \& Watson, S. E. (1995). Object-based attentional selection: Grouped-arrays or spatially-invariant representations? Journal of Experimental Psychology: General, 126, 3-13.

KrösE, B. J., \& JULESZ, B. (1989). The control and speed of shifts of attention. Vision Research, 29, 1607-1619.

LaBerge, D. (1983). Spatial extent of attention to letters and words. Journal of Experimental Psychology: Human Perception \& Performance, 9, 371-379.

LABERGE, D. (1995). Attentional processing: The brain's art of mindfulness. Cambridge, MA: Harvard University Press.

LAVIE, N. (1995). Perceptual load as a necessary condition for selective attention. Journal of Experimental Psychology: Human Perception \& Performance, 21, 451-468.

Lavie, N., \& TSAL, Y. (1994). Perceptual load as a major determinant of the locus of selection in visual attention. Perception \& Pspchophysics, 56, 183-197.

Luck, S. J., Chelazzi, L., Hillyard, S. A., \& Desimone, R. (1997).
Neural mechanisms of spatial selective attention in areas $\mathrm{V} 1, \mathrm{~V} 2$, and $\mathrm{V} 4$ of macaque visual cortex. Journal of Neurophysiology, 77, 24-42.

Luck, S. J., Fan, S., \& Hillyard, S. A. (1993). Attention-related modulation of sensory-evoked brain activity in a visual search task. Journal of Cognitive Neuroscience, 5, 188-195.

LuCK, S. J., \& HilLyaRD, S. A. (1994a). Electrophysiological correlates of feature analysis during visual search. Psychophysiology, 31, 291-308.

LuCK, S. J., \& HillyaRD, S. A. (1994b). Spatial filtering during visual search: Evidence from human electrophysiology. Journal of Experimental Psychology: Human Perception \& Performance, 20, 1000-1014.

Mangun, G. R., \& Hillyard, S. A. (1988). Spatial gradients of visual attention: Behavioral and electrophysiological evidence. Electroencephalography \& Clinical Neurophysiology, 70, 417-428.

MiLliken, B., TipPER, S. P., \& WEAVER, B. (1994). Negative priming in a spatial localization task: Feature mismatching and distractor inhibition. Journal of Experimental Psychology: Human Perception \& Performance, 20, 624-646.

Moran, J., \& Desimone, R. (1985). Selective attention gates visual processing in the extrastriate cortex. Science, 229, 782-784.

MOTtER, B. C. (1993). Focal attention produces spatially selective processing in visual cortical areas V1, V2, and V4 in the presence of competing stimuli. Journal of Neurophysiology, 70, 909-919.

MOTrER, B. C. (1994). Neural correlates of attentive selection for color or luminance in extrastriate area V4. Journal of Neuroscience, 4 , 2178-2189

Mozer, M. C. (1991). The perception of multiple objects. Cambridge, MA: MIT Press.

NEATH, I., \& TARR, M. J. (1993). The allocation of visual attention across noncontiguous spatial locations. Unpublished manuscript.

Olshausen, B., Anderson, C., \& Van Essen, D. (1993). A neural model of visual attention and invariant pattern recognition based on dynamic routing information. Journal of Neuroscience, 13, 4700-4719.

PARK, J., \& KANWISHER, N. (1994). Negative priming for spatial locations: Identity matching, not distractor inhibition. Journal of Experimental Psychology: Human Perception \& Performance, 20, 613-623.

POSNER, M. I., \& COHEN, Y. (1984). Components of visual orienting. In H. Bouma \& D. Bowhuis (Eds.), Attention and performance $X$ (pp. 531-556). Hillsdale, NJ: Erlbaum.

Posner, M. I., NisSEN, M. J., \& Ogden, W. C. (1978). Attended and unattended processing modes: The role of set for spatial location. In H. J. Pick \& I. J. Saltzman (Eds.), Modes of perception (pp. 137-157). Hillsdale, NJ: Erlbaum

Posner, M. I., SNyder, C. R. R., \& Davidson, B. J. (1980). Attention and the detection of signals. Journal of Experimental Psychology: General, 109, 160-174.

Rizzolatti, G., Riggio, L., Dascola, I., \& Umiltà, C. (1987). Reorienting attention across the horizontal and vertical meridians: Evidence in favor of a premotor theory of attention. Neuropsychologia, 25, 31-40.

RuthrufF, E., \& Miller, J. (1995). Negative priming depends on ease of selection. Perception \& Psychophysics, 57, 715-723.

SAGI, D., \& JULESZ, B. (1986). Enhanced detection in the aperture of focal attention during simple discrimination tasks. Nature, 321, 693-695.

Schall, J. D., Hanes, D. P., Thompson, K. G., \& King, D. J. (1995). Saccade target selection in frontal eye field of Macaque. I. Visual and premovement activation. Journal of Neuroscience, 15, 6905-6918.

Shapiro, K. L., \& Loughlin, C. (1993). The locus of inhibition in the priming of static objects: Object token vs. location. Journal of Experimental Psychology: Human Perception \& Performance, 19, 352-363.

Shulman, G. L., Remington, R. W., \& MCLean, J. P. (1979). Moving attention through physical space. Journal of Experimental Psychology: Human Perception \& Performance, 5, 522-526.

Sperling, G., \& WeichSelgartner, E. (1995). Episodic theory of the dynamics of spatial attention. Psychological Review, 102, 503-532.

Theeuwes, J. (1991). Cross-dimensional perceptual selectivity. Perception \& Psychophysics, 50, 184-193.

Theeuwes, J. (1992). Perceptual selectivity for color and form. Perception \& Psychophysics, 51, 599-606.

TheEUWES, J. (1994). Stimulus-driven capture and attentional set: Selective search for color and visual abrupt onsets. Journal of Experimental Psychology: Human Perception \& Performance, 20, 799-806. 
Theeuwes, J. (1995). Temporal and spatial characteristics of preattentive and attentive processing. Visual Cognition, 2, 221-233.

THeeuwes, J. (1996). Perceptual selectivity for color and form: On the nature of the interference effect. In A. F. Kramer, M. G. H. Coles, \& G. D. Logan (Eds.), Converging operations in the study of visual selective attention (pp. 297-314). Washington, DC: American Psychological Association.

Thompson, K. G., Bichot, N. P., \& Schall, J. D. (1997). Dissociation of visual discrimination from saccade programming in macaque frontal eye field. Journal of Neurophysiology, 77, 1046-1050.

Thompson, K. G., HaNes, D. P., Bichot, N. P., \& Schall, J. D. (1996). Perceptual and motor processing stages identified in the activity of macaque frontal eye field neurons during visual search. Journal of Neurophysiology, 76, 4040-4055.

Tipper, S., Brehaut, J. C., \& Driver, J. (1990). Selection of moving and static objects for the control of spatially directed attention. Journal of Experimental Psychology: Human Perception \& Performance, $3,492-504$.

TiPPER, S. P., \& CRANSTON, M. (1985). Selective attention and priming: Inhibitory and facilitatory effects of ignored primes. Quarterly Journal of Experimental Psychology, 37A, 591-611.

TIPPER, S. P., Weaver, B., \& Milliken, B. (1995). Spatial negative priming without mismatching: Comment on Park and Kanwisher (1994). Journal of Experimental Psychology: Human Perception \& Performance, 21, 1220-1229.

Treisman, A. M. (1988). Features and objects: The Fourteenth Bartlett Memorial Lecture. Quarterly Journal of Experimental Psychology, 40A, 201-237.

Treisman, A. M., \& Sato, S. (1990). Conjunction search revisited. Journal of Experimental Psychology: Human Perception \& Performance, 16, 459-478.

Treue, S., \& Maunsell, J. H. R. (1996). Attentional modulation of visual motion processing in cortical areas MT and MST. Nature, 382, 539-541.
TSAL, Y. (1983). Movements of attention across the visual field. Journal of Experimental Psychology: Human Perception \& Performance, 9, 523-530.

Tsal, Y., \& LAvie, N. (1988). Attending to color and shape: The special role of location in selective visual processing. Perception \& Psychophysics, 44, 15-21.

TSAL, Y., \& LAVIE, N. (1993). Location dominance in attending to color and shape. Journal of Experimental Psychology: Human Perception \& Performance, 19, 131-139.

WOLFE, J. M. (1994). Guided Search 2.0: A revised model of visual search. Psychonomic Bulletin \& Review, 1, 202-238.

WOLFE, J. M., \& POKORNY, C. W. (1990). Inhibitory tagging in visual search: A failure to replicate. Perception \& Psychophysics, 48, 357-362.

Worden, M., Schneider, W., \& Wellington, R. (1995, November). Assessing the distribution of exogenous and endogenous attentional modulation in human visual cortex with $f M R I$. Paper presented at the meeting of the Psychonomic Society, Los Angeles.

YANTIS, S. (1988). On analog movements of visual attention. Perception \& Psychophysics, 43, 203-206.

Zimba, L. D., \& Hughes, H. C. (1987). Distractor-target interactions during directed visual attention. Spatial Vision, 2, 117-149.

\section{NOTE}

1. The probe RTs for digit locations could have been categorized either according to horizontal and vertical midline crossings or according to distance of each midline from the target location. Given the similarity between horizontal and vertical midlines in Hughes and Zimba's (1985, 1987) and Rizzolatti et al.'s (1987) findings, we chose to categorize by distance of the midline from the target.

(Manuscript received February 8, 1996; revision accepted for publication July 24,1997 .) 